\title{
Volume Magnetization for System-Level Testing of Magnetic Materials within Small Satellites
}

\author{
David T. Gerhardt ${ }^{1}$, Scott E. Palo \\ University of Colorado at Boulder, Aerospace Engineering Sciences \\ Boulder, CO 80303, U.S.A.
}

\begin{abstract}
Passive Magnetic Attitude Control (PMAC) is a popular among small satellites due to its low resource cost and simplicity of installation. However, predicting the performance of these systems can be a challenge, chiefly due to the difficulty of measurement and simulation of hysteresis materials. We present a low-cost method of magnetic measurement allowing for characterization of both hard and soft magnetic materials. A Helmholtz cage uniformly magnetizes a $30 \mathrm{~cm} \times 30 \mathrm{~cm} \times 30 \mathrm{~cm}$ test volume. The addition of a thin sense coil allows this system to characterize individual hysteresis rod performance when in close proximity to other hard and/or soft magnetic materials. This test setup is applied to hard and soft magnetic materials used aboard the Colorado Student Space Weather Experiment (CSSWE), a 3U CubeSat for space weather investigation which used a PMAC system. The measured hard magnet dipole of $0.80 \pm 0.017 \mathrm{~A} \cdot \mathrm{m}^{2}$ is in good agreement with the dynamics-based satellite dipole moment fits. Five hysteresis rods from the same set as the CSSWE flight rods are tested; significant differences in dampening abilities are found. In addition, a limitation of the widely-used Flatley model is described. The interaction of two hysteresis rods in a variety of relative geometries are tested; perpendicular rods are found to have no significant interaction while parallel rods could have their dampening ability reduced by half, depending on the rod separation distance. Finally, the performance of the hysteresis rods are measured in their flight configuration, with hard and soft magnetic material dispersed as it is on CSSWE itself. For the CSSWE PMAC system design, interactions between rods have a greater affect than
\end{abstract}

\footnotetext{
${ }^{1}$ Email address: dtgerhardt@gmail.com Phone: +45 31452161
} 
the magnetic flux density offset due to the onboard bar magnet.

Keywords: Passive Magnetic, Attitude Control, PMAC, Hysteresis, Magnetic Measurement, CSSWE

The recent surge in small satellites has caused a renewed interest in simple, low-resource attitude control methods. One such method is Passive Magnetic Attitude Control (PMAC), which uses a combination of hard and soft magnetic materials to align a satellite with the earth's local magnetic field. First used in 1960 [1], PMAC continues to be a useful attitude control method, especially for both student [2], [3] and science investigation [4] [5] nanosatellites.

A key example of a PMAC satellite is the Colorado Student Space Weather Experiment (CSSWE) [6]; [7]; [8]. Developed at the University of Colorado $(\mathrm{CU}), \mathrm{CSSWE}$ is a $3 \mathrm{U}(10 \mathrm{~cm} \times 10 \mathrm{~cm} \times 30 \mathrm{~cm})$ CubeSat built to study space weather. As part of the NASA ELaNa VI launch on September 13, 2012, CSSWE was inserted into a $478 \mathrm{~km} \times 786 \mathrm{~km}, 64.7^{\circ}$ inclination orbit, along with 10 other CubeSats [9]; [10]. CSSWE successfully aligned within $15^{\circ}$ of the local magnetic field after one week on-orbit [11]. CSSWE extended its science mission to 438 days, nearly $5 \times$ its full mission success duration.

Although PMAC is popular, it is difficult to predict its performance. Some nanosatellites have taken as long as two months to align to the local magnetic field, when they were expected to align within days or orbits [12] [13]. Excessive dampening times can have a critical impact on attitudedependent science operations, especially for nanosatellites which tend to have short mission lifetimes. A critical aspect of accurate PMAC performance prediction is characterization of the magnetic materials used in the system.

As the old adage says, "garbage in equals garbage out"; inaccurate input translates to inaccurate output. The key inputs to PMAC simulation are magnetic torques; thus the parameters which govern these torques must be accurately measured to ensure that the simulation results are trustworthy. We describe measurement techniques to accurately predict the magnetic moments of hard and soft magnetic materials. In a typical PMAC system, this relates to the magnetic moment of the bar magnet $\mathbf{m}$ and the hysteresis rods.

First, the characteristics of a Helmholtz cage useful for nanosatellite magnetic measurements are described (Section 1). Next, the static magnet magnetic moment $\mathbf{m}$ is measured (Section 2). A Helmholtz-cage-based hysteresis rod measurement method is described (Section 3); this method is applied 
to characterize the rod performance both with and without other magnetic materials in proximity.

\section{Helmholtz Cage}

A Helmholtz cage [14] is a set of six wire coils (two per orthogonal axis) which can provide an arbitrary uniform field within the volume enclosed by the coils; the properties of the uniform field depend upon the cage dimensions and available current. The cage operates on the principle that a current-carrying wire will produce a magnetic field, and can be used to both cancel the inherent magnetic field and supply an arbitrary magnetic field. A Helmholtz cage was constructed to perform multiple magnetic measurements relevant to a PMAC system. The theory, design trades, and construction of nanosatellite Helmholtz cages are well-documented in established litera-

ture [15], [16]; thus only the characteristics and validation tasks are presented here.

\subsection{Characteristics}

The Helmholtz cage was designed for the testing of a CubeSat spacecraft; the following requirements govern its design:

1. The Helmholtz cage test volume shall have dimensions of $30 \mathrm{~cm} \times 30 \mathrm{~cm}$ $\times 30 \mathrm{~cm}$.

2. The Helmholtz cage test volume shall provide $1 \%$ theoretical field uniformity along each axis.

3. The Helmholtz cage shall provide a magnetic field strength range of $\pm 50 \mathrm{~A} / \mathrm{m}$ on each axis.

The largest standard CubeSat is a $3 \mathrm{U}$ size, with dimensions $10 \mathrm{~cm} \times 10 \mathrm{~cm}$ $\times 34 \mathrm{~cm}$ [17]. Thus, requirement 1 ensures that the test volume is sufficient to measure any standard CubeSat (a 3U CubeSat easily fits when diagonally placed). Requirement 2 defines the expected uniformity of each axis of the Helmholtz cage. It is given in terms of theory because the empirical uniformity of each axis which may be measured by a magnetometer is dependent on the component of the earth field along that axis. If the Helmholtz cage were placed in a magnetically noisy or non-uniform environment, the output field of the cage would be similarly noisy. However, a benefit of the small test volume is portability. If an environment is particularly noisy or nonuniform, the cage may be moved to a location that possesses a more stable 
Table 1: The characteristics of the as-built Helmholtz cage are shown.

\begin{tabular}{lll}
\hline Characteristic & Value & Unit \\
\hline Coil Geometry & Square & - \\
Coil Side Length & 62.25 & $\mathrm{~cm}$ \\
Coil-to-Coil Spacing & 37.24 & $\mathrm{~cm}$ \\
Windings per coil & 30 & turns \\
Magnet Wire & 22 & $\mathrm{AWG}$ \\
Magnetizing Field (per axis) & \pm 100 & $\mathrm{~A} / \mathrm{m}$ \\
\hline
\end{tabular}

field. Requirement 3 defines the configurable field within the Helmholtz cage after nullifying the earth-based local magnetic field.

The requirements are met by the Helmholtz cage design given in Table 1 . The theoretical field uniformity for the as-built Helmholtz cage is shown in Figure 1. The Helmholtz cage design meets all design requirements.

Figure 2 shows the support hardware and connections necessary for operation of the Helmholtz cage. A LabView software interface allows the user to set a desired constant arbitrary field within the Helmholtz cage. This highlevel task is performed by using a PIC18F452 microcontroller to read the magnetic field from a calibrated PNI MicroMag 3-axis magnetometer, and controlling the field via the combination of a programmable power supply and a relay bank, which allows each axis of the cage to have an independently-set positive or negative current flow within $10 \mathrm{~mA}$.

\subsection{Characterization}

Thus, the planar field of the Helmholtz cage was mapped by measuring the total magnetic flux density at each point of a $5 \mathrm{~cm}$ grid. This mapping was performed twice: once with no current through the Helmholtz coils and once with the Helmholtz cage set to nullify the local field. Figures $3 \mathrm{a}$ and $3 \mathrm{~b}$ show the magnetic flux density magnitude as a function of planar position. At the time and location of the test, the earth field varied by $5 \mu$ Tesla over the test area. This caused some variation in the zeroed magnetic field, as shown in Figure 3b. As shown, when the Helmholtz cage is zeroed, the test area magnetic field takes the shape of a bowl with a square base: the $\pm 10 \mathrm{~cm}$ central area has a measured uniformity of $2 \mu$ Tesla, but the field near the edges of the test area reaches $10 \mu$ Tesla. The measured field strength variation after field nullification is $5 \%$; this is roughly equivalent to the local 


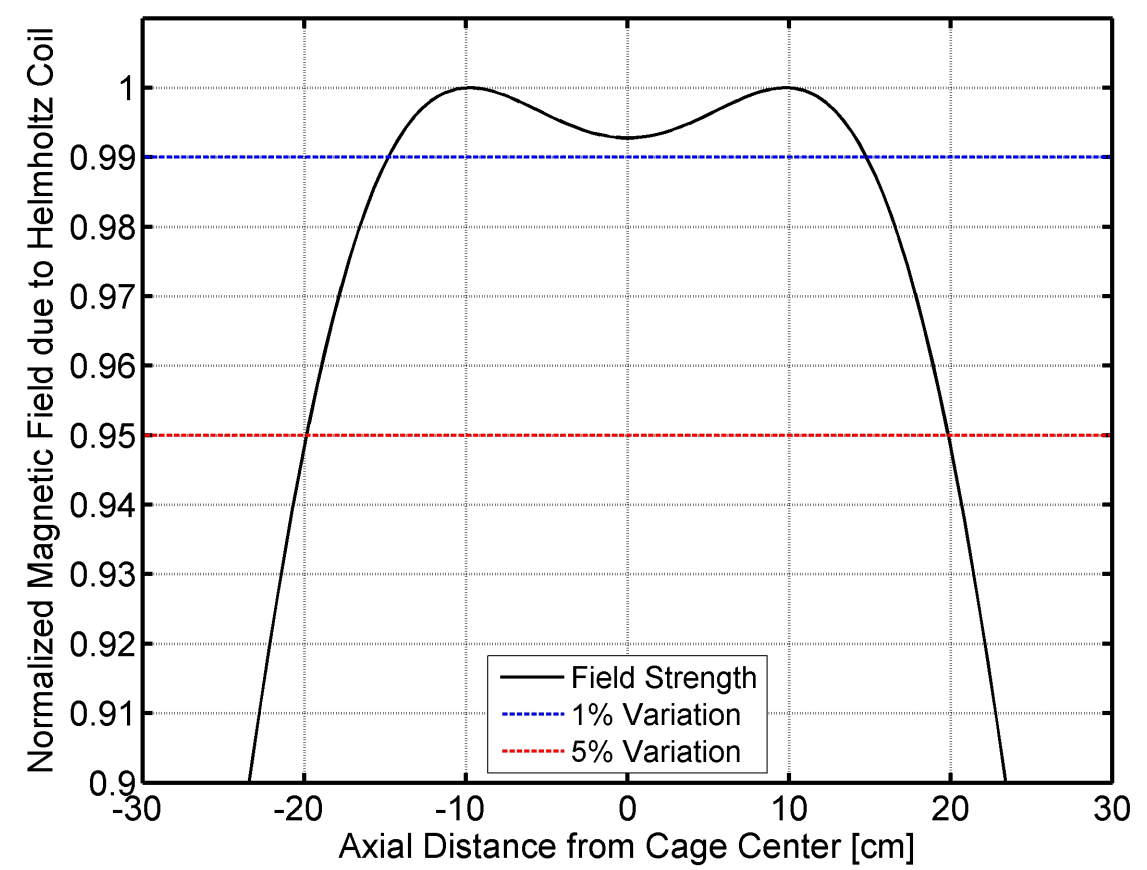

Figure 1: The theoretical axial magnetic field of the as-built Helmholtz cage with a spacing of $h=1.2 a=37.24 \mathrm{~cm}$. The magnetic flux density $B$ is normalized by the maximum flux density. 


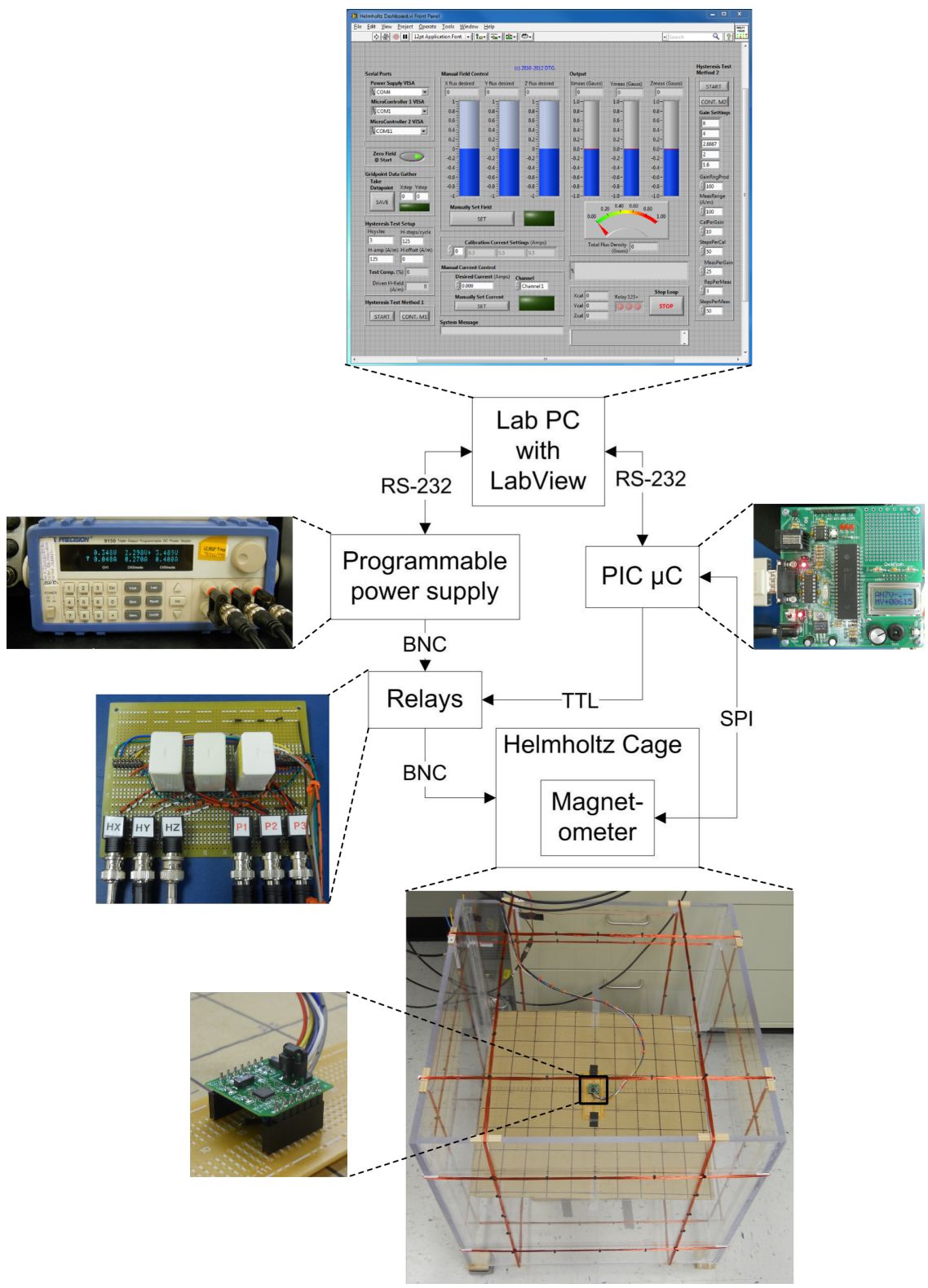

Figure 2: Helmholtz hardware chain showing how user-set values of magnetic field on each axis are converted to the appropriate current through the Helmholtz coils. 
field variation before nullification. Repeating the test in a more uniform local field may yield better results. However, a $20 \mathrm{~cm} \times 20 \mathrm{~cm} \times 20 \mathrm{~cm}$ test volume maintained at 0-2 $\mu$ Tesla is sufficient for the magnetic testing described later in this paper.

\section{Bar Magnet Measurement}

The Helmholtz cage was used to measure the magnetic moment of the bar magnet chosen for use in CSSWE. The bar magnet moment is important because it is directly responsible for the magnetic torque generated upon orbit. The magnetic torque due to any magnetic dipole in a local field is as follows:

$$
\mathbf{L}=\mathbf{m} \times \mathbf{B}_{\text {earth }}
$$

where $\mathbf{m}$ is a magnetic dipole vector and $\mathbf{B}_{\text {earth }}$ is the local magnetic flux density vector due to earth. During simulation, the inertial local magnetic field vector due to earth $\mathbf{B}_{\text {earth }}$ may be sufficiently predicted using the International Geomagnetic Reference Field (IGRF-11) model; only the satellite position is necessary as an input [18]. The dipole vector $\mathbf{m}$ could be due to hard or soft magnetic material. In the case of the bar magnet, the dipole vector is hard and does not change with orientation. Thus, the bar magnet dipole may be measured on the ground to obtain a good estimate of its on-orbit performance.

The steps for the bar magnet measurement are given below:

1. Ensure bar magnet is far from Helmholtz cage.

2. Take measurement of local magnetic field while magnetometer is at the center of the Helmholtz cage.

3. Supply current to the Helmholtz coils to null the magnetic field on all three axes. Record the power supply current provided to each axis.

4. Using the $5 \mathrm{~cm}$ analysis grid, move the PNI MicroMag 3-axis magnetometer, measuring the $B$-field magnitude in the $30 \mathrm{~cm} \times 30 \mathrm{~cm}$ area defined by the grid in steps of $5 \mathrm{~cm}$.

5. Place the bar magnet in the center of the analysis grid.

6. Again using the analysis grid, record the $B$-field magnitude at the same positions as in step (4). Ignore grid positions less than $8 \mathrm{~cm}$ from the bar magnet position as this may saturate the magnetometer and the dipole model is more accurate with greater distance from the dipole. 


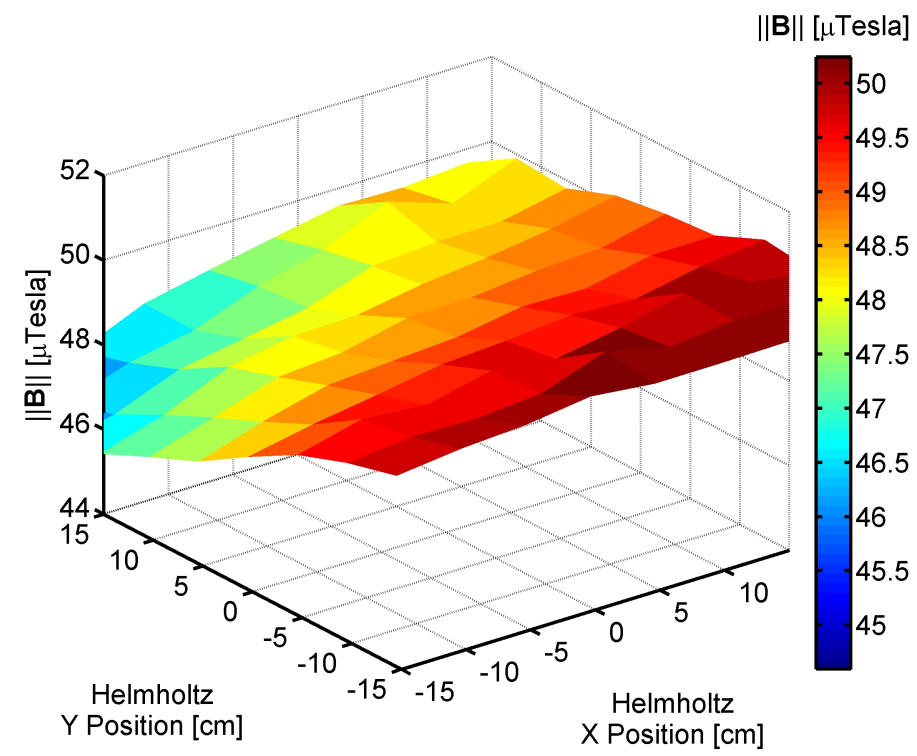

$\|\mathbf{B}\|[\mu \mathrm{Tesla}]$

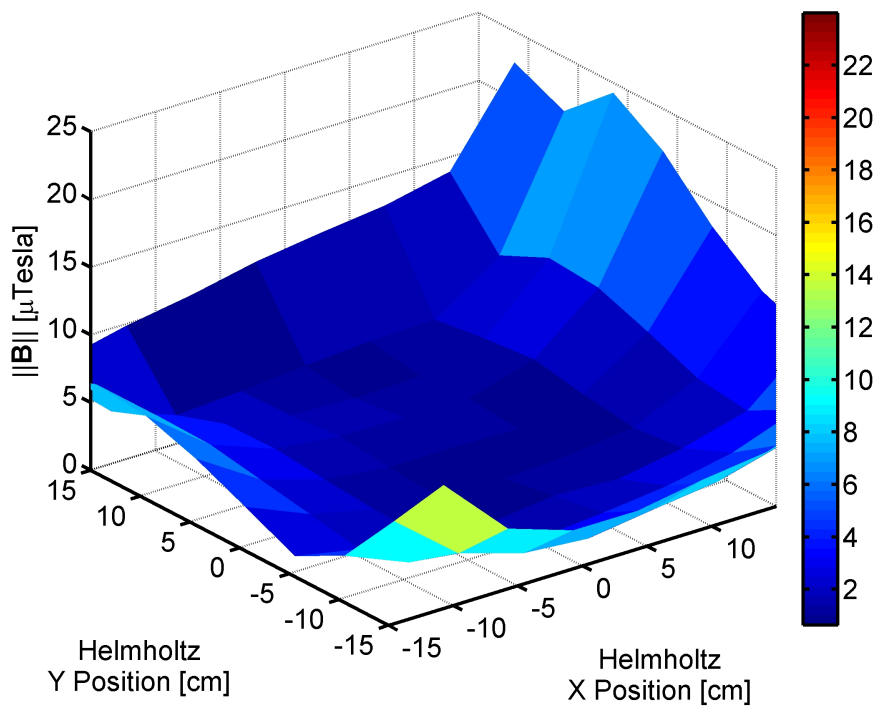

Figure 3: The magnetic flux density magnitude of the Helmholtz cage $30 \mathrm{~cm} \times 30 \mathrm{~cm}$ test area is shown with $5 \mathrm{~cm}$ resolution. The upper plot (a) shows the distribution within the cage when there is no current in the coils; the lower plot (b) shows the distribution when the cage is set to null the magnetic field. Note the change in $B$-field scaling between a and $\mathrm{b}$. 
Once the two datasets are recorded and corrected for magnetometer calibration error, the $B$-field magnitude at each grid position due to the bar magnet alone may be calculated by subtracting the no-bar-magnet data from the bar-magnet data. The measured magnetic flux density magnitude $\|\mathbf{B}\|_{\text {measured }}$ data are fit to the magnetic dipole magnitude formula using a non-linear least squares fit. The fitted magnetic dipole magnitude equation is $[19]$ :

$$
\|\mathbf{B}\|_{\text {measured }}=\left(\frac{\mu_{0} m_{\mathrm{fit}}}{4 \pi r^{3}}\right) \sqrt{1+3 \cos ^{2} \nu}
$$

where $m_{\text {fit }}$ is the fitted magnetic moment, $r$ is the distance from the magnet to the measurement location, and $\nu$ is the magnetic co-latitude of the measurement location $\left(90^{\circ}\right.$ represents a magnetometer position co-planar with the bar magnet position). Three independent measurements were performed, yielding a bar magnet magnetic moment $\|\mathbf{m}\|=0.80 \pm 0.017 \mathrm{~A} \cdot \mathrm{m}^{2}$ after a small sample size correction to the standard error of the mean has been applied [20]. Figure 4 shows the magnitude of the magnetic flux density and the magnetic dipole fit for the bar magnet measurement data for one of these measurements.

\section{Hysteresis Measurement}

The crux of a PMAC system is the hysteresis dampening. It is the most difficult torque to predict and can greatly affect the system settling time. The goal of hysteresis rod magnetic measurement is to determine the relationship between the earth-based $H$-field and the magnetic torque due to the hysteresis rods. As shown in Equation 1, the hysteresis torque may be calculated if the hysteresis-based magnetic dipole is known. However, the hysteresis material is soft; its magnetic dipole changes as its orientation with respect to the magnetic field changes. The magnetic moment parallel to a hysteresis rod may be defined as [21]:

$$
m_{\text {hyst }}=V\left(\frac{B_{\text {hyst }} / \mu_{0}-H_{a}}{1-N_{d}}\right)
$$

where $V$ is the volume of the hysteresis rod, $\mu_{0}$ is the permeability of free space, $B_{\text {hyst }}$ is the average parallel magnetic flux density within the rod, $H_{a}$ is the applied field parallel to the rod, and $N_{d}$ is the demagnetization factor. Many groups $([22],[2],[23],[3],[24])$ simplify Equation 3 to $m_{\text {hyst }}=V B / \mu_{0}$. It 


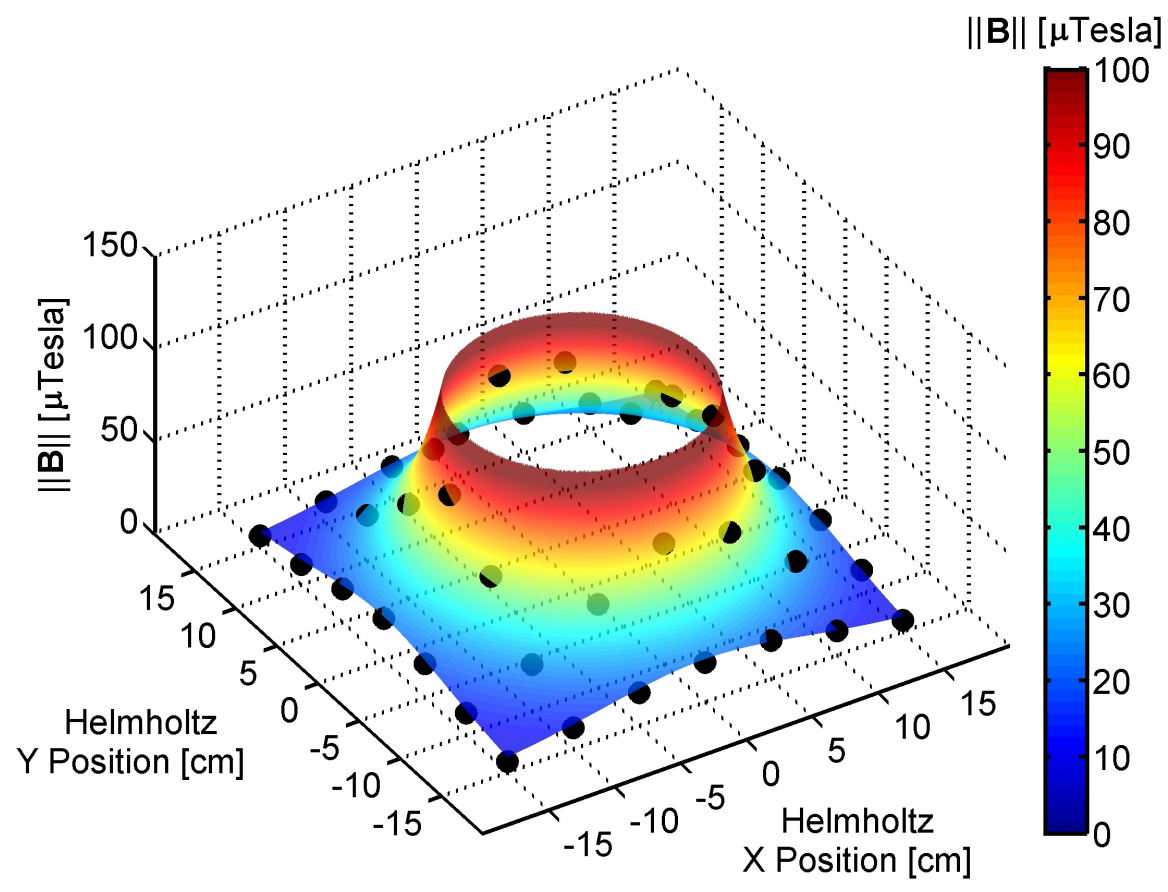

Figure 4: A single bar magnet measurement dataset with the fitted $0.80 \mathrm{~A} \cdot \mathrm{m}^{2}$ magnetic dipole overlaid. The $B$-field data as recorded by the PNI MicroMag 3-axis magnetometer are shown in black. The magnetic dipole field is shown using the colored surface plot. The dipole field within $8 \mathrm{~cm}$ of the origin is omitted for clarity. This dataset has an RMS error of $3.96 \mu \mathrm{T}$ relative to the $0.80 \mathrm{~A} \cdot \mathrm{m}^{2}$ fit. 
is feasible to ignore $N_{d}$ in the denominator for rods with a length-to-diameter radio $L / D>30$ [21], as the error is $<2 \%$ (the demagnetizing field has not been ignored, it is taken into account by emperically measuring $B$ ). The $H_{a}$ term may be ignored if $B_{\text {hyst }} / \mu_{0} \gg H_{a}$ (true for most properly-designed PMAC hysteresis rods).

As shown via Equations 1 and 3, the hysteresis torque may be predicted if there is a relation between the magnetic field parallel to the hysteresis rod $H_{a}$ and the average magnetic flux density within the hysteresis rod $B_{\text {hyst }}$. This section seeks to measure this non-linear relationship between $H_{a}$ and $B_{\text {hyst }}$, often called the hysteresis loop.

The use of an excitation (magnetizing) \& sense (or pickup) coil is an accepted method of hysteresis measurement [21]. Recent empirical testing of hysteresis rods for PMAC systems have been performed by two main groups: Ivanov et al. [25] (also [26]) use a small excitation solenoid (only magnetizing one hysteresis rod), while Santoni et al. [27] (also [28], [29]) use a larger excitation solenoid capable of magnetizing a large array of rods. We present a method of using the Helmholtz cage itself as the excitation coil, as it is able to supply a uniform magnetizing field in a large volume and in any direction. This allows multiple samples to be magnetized simultaneously to test their coupled magnetic performance while reusing hardware that may already be available to nanosatellite developers. Some PMAC satellites have had significantly delayed settling times ([12], [13]); ignoring the system-level magnetic coupling may have had a large role in these delays.

\subsection{Theory}

A sense coil connected to an integrator is capable of directly measuring the average interior magnetic flux density of a sample. The Helmholtz cage enables the user to apply a time-varying magnetizing field over a large test volume. The addition of a sense coil to the Helmholtz cage results in a setup which is capable of system-level measurements at a nanosatellite scale.

The theory begins with Faraday's law, which states that a wire coil shall have a voltage generated within it proportional to the rate of change of magnetic flux through the coil [21]:

$$
\mathcal{E}=-N \frac{d \phi}{d t}
$$

where $\mathcal{E}$ is the voltage induced in the coil, $N$ is the number of turns of the search coil, $\phi$ is the magnetic flux in SI units of weber $\left(1 \mathrm{~Wb}=1\right.$ Tesla $\left.\cdot \mathrm{m}^{2}\right)$, 
and $t$ is time. Note that voltage is only induced by a changing magnetic flux through the coil. Equation 4 may be rearranged and integrated:

$$
\int_{0}^{t} \mathcal{E} d t=-N \int_{\phi_{1}}^{\phi_{2}} d \phi=-N \Delta \phi
$$

where $\Delta \phi$ represents the change in $\phi$. Because the magnetic flux is directly related to the magnetic flux density by the area of the search coil $A$, Equation 5 can be written in terms of magnetic flux density:

$$
\int_{0}^{t} \mathcal{E} d t=-N A \Delta B
$$

The integration in Equation 6 may be carried out one of two ways: through hardware or software. The hardware method uses a typical opamp integrator circuit. Commercial versions of this circuit (sometimes called a "fluxmeter") are available for purchase but they were found to be prohibitively expensive. The hardware integrator circuit behaves as follows [21]:

$$
\mathcal{E}_{\text {out }}=(R C)^{-1} \int_{0}^{t} \mathcal{E}_{\text {in }} d t
$$

where $R$ is the resistor value and $C$ is the capacitor value. Thus, for a sense coil in series with the integrator:

$$
\mathcal{E}_{\text {out }}=-\left(\frac{N A}{R C}\right) \Delta B
$$

The values of the resistor and capacitor may be calculated based on the materials to be measured. However, after building a hardware-based integrator, we found that temperature- and offset-voltage-based drift made measurements with the hardware integrator difficult. This drift caused the measured hysteresis loops to be significantly distorted and unsuitable for fitting with a hysteresis model. The hardware could be calibrated for one measurement at a time, but the system would drift too much over the course of multiple measurements to be useful for fitting purposes. A commercial fluxmeter is expensive partially because it is built to correct for these drifts. The implemented low-cost solution is to perform the integration digitally.

The recently published paper by Ivanov et al. [25] finds that analog integrators properly sized to avoid attenuation errors do not have sufficient signal 
levels to accurately measure the properties of nanosatellite-sized PMAC hysteresis rods; digital integration is preferable. However, even with digital integration, the input voltage must be high enough to be read by the ADC without excessive quantization errors. Equation 4 shows that the voltage out of the sense coil is directly related to the frequency of the magnetizing field. Ivanov et al. [25] shows that eddy currents can cause the measured hysteresis properties to be in error if sensed at high frequencies. For this reason, Ivanov chooses a magnetizing field frequency of $0.2 \mathrm{~Hz}$. We have chosen a magnetizing field frequency of $1 \mathrm{~Hz}$ as a good balance between errors caused by ADC quantization and errors caused by the eddy currents.

\subsection{Setup}

Figure 5 is a block diagram of the hardware setup for hysteresis measurement using the Helmholtz cage and a sense coil. Each element of the hardware setup is detailed below.

\subsubsection{Sense Coil}

The magnet wire cannot be wrapped around the hysteresis rod itself due to the minimum bend radius of the magnet wire and the risk of bending the hysteresis rod (thus damaging its magnetic properties). Instead, a sense coil is built such that magnetic samples can be slid within the coil when desired. In order to measure the average B-field of the magnetic sample, the sense coil length should extend the length of the sample. In this case, the sample is the hysteresis rod with a length of $97.17 \pm 0.03 \mathrm{~mm}$ and cross-sectional area $A_{m}=0.805 \pm 0.00064 \mathrm{~mm}^{3}$. The sense coil is built by tightly winding 36 AWG magnet wire (manufacturer-listed diameter of $0.1400 \mathrm{~mm} \pm 0.0013$ ) around a nonmagnetic aluminum tube with an inner/outer diameter of $5.00 / 5.30 \pm 0.03 \mathrm{~mm}$ which yields a cross-sectional sensing area $A_{s}=21 \pm 1.8 \mathrm{~mm}^{2}$. The as-built sense coil (shown within the Helmholtz cage in Figure 6) has a wire-wrapped length of $96.48 \pm 0.03 \mathrm{~mm}$; the number of turns is calculated to be $N=691 \pm 6$.

Because the sense coil is wrapped around a hollow tube, the magnetic flux picked up by the sense coil is due to both the magnetic flux through the magnetic material and the magnetic flux through the air surrounding it (yet still within the coil). Thus, the magnetic flux density of each measurement is corrected as follows: [21]

$$
B_{\text {true }}=B_{\text {apparent }}-\mu_{0} H_{a}\left(\frac{A_{s}-A_{m}}{A_{m}}\right)
$$




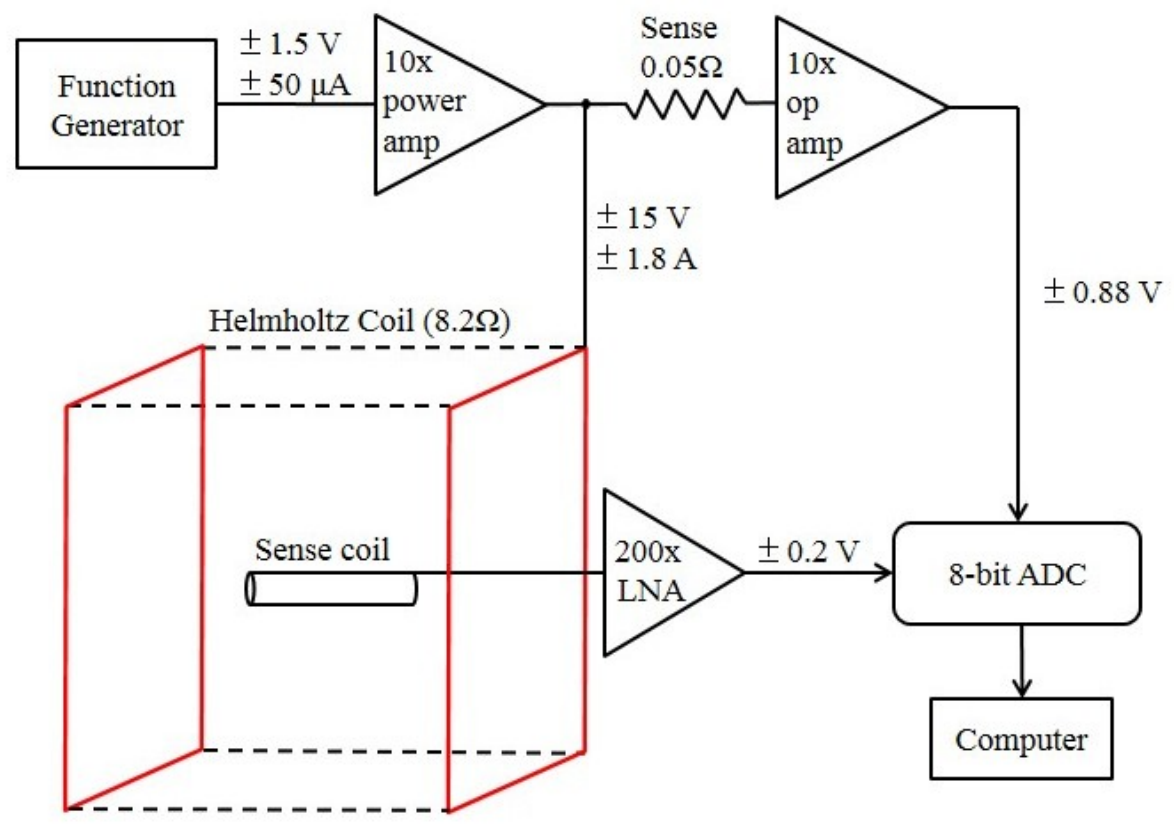

Figure 5: The hysteresis rod measurement hardware setup block diagram. The labeled voltages and currents are for $\mathrm{H}$-field cycling at $\pm 100 \mathrm{~A} / \mathrm{m}$ amplitude; the amplitude (and subsequent voltages and currents) may be decreased as desired. 


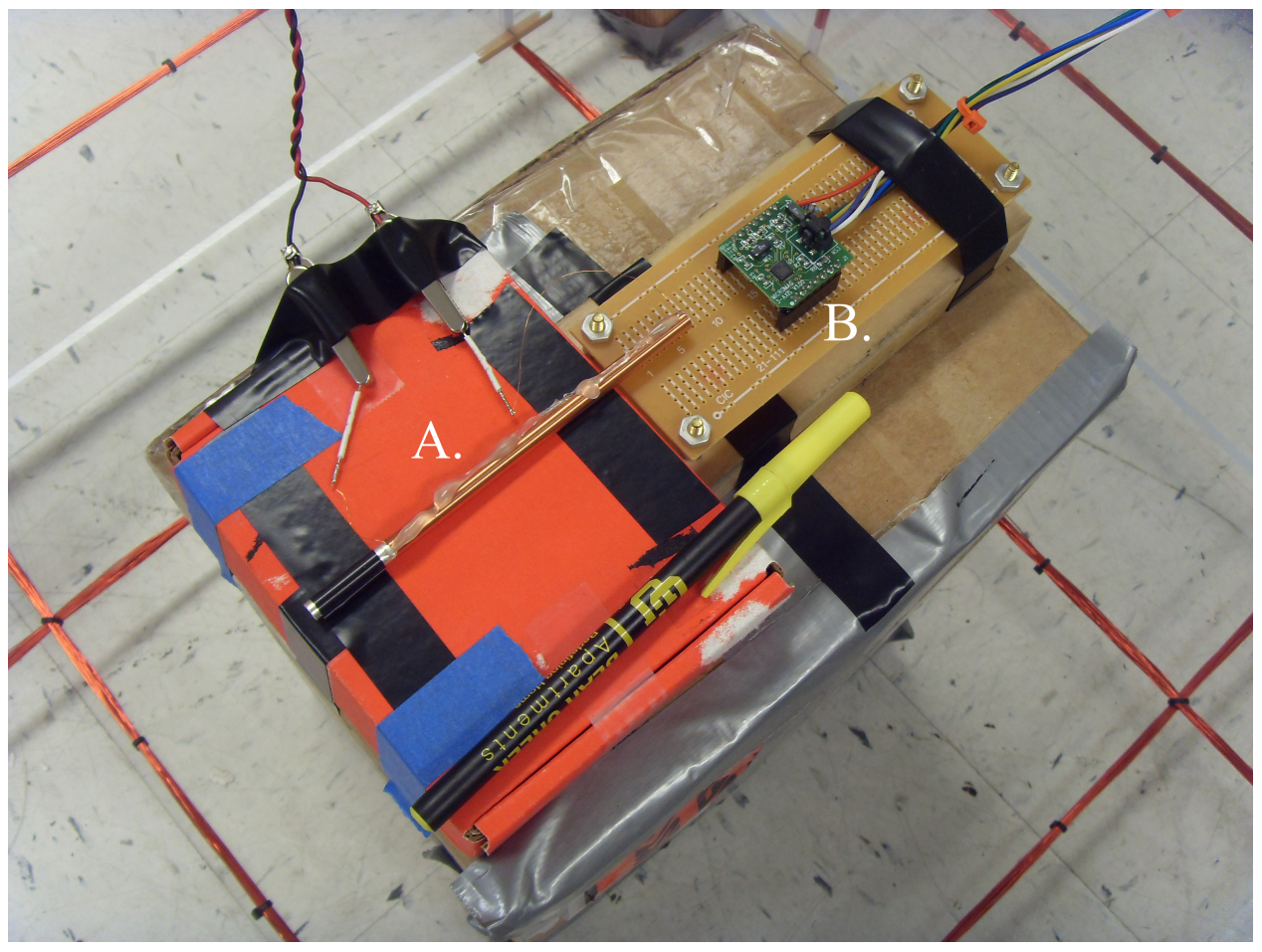

Figure 6: The finished hysteresis measurement sense coil (A.) with approximately 691 turns of 36 AWG wire. A pen is shown next to the sense coil for length scale reference; the MicroMag3 3-axis magnetometer (B.) is also visible. 
where $B_{\text {apparent }}$ is the magnetic flux density as measured via Equation $6, H_{a}$ is the applied field, $A_{s}$ is the cross-sectional area of the sense coil, and $A_{m}$ is the cross-sectional area of the magnetic material.

\subsubsection{Helmholtz Cage}

The Helmholtz cage is used to provide the changing magnetizing field strength which causes the magnetic flux density within a magnetic sample to vary. The Helmholtz cage and support equipment is capable of controlling the static magnetizing field (including field nullification) in steps of $0.59 \pm 0.059$ $\mathrm{A} / \mathrm{m}$.

The Helmholtz cage is small enough to allow for orientation changes which can result in improved performance. Before hysteresis rod testing begins, the Helmholtz cage is oriented such that the X-axis is perpendicular to the magnetic field. This allows for testing over the full $\pm 100 \mathrm{~A} / \mathrm{m}$ magnetizing field range which the Helmholtz cage can produce.

\subsubsection{Other Hardware}

The Agilent 33220A function generator is used to generate a $1 \mathrm{~Hz}$ sine wave with an amplitude of up to $\pm 1.5 \mathrm{~V}$ for a desired $\mathrm{H}$-field of $\pm 100 \mathrm{~A} / \mathrm{m}$. This low-current signal is fed to an Apex PA16 power operational amplifier, which multiplies the voltage by a factor of ten while acting as a current source for a single coil set of the Helmholtz cage. The maximum output current of $\pm 1.8 \mathrm{~A}$ supplies an H-field of about $\pm 100 \mathrm{~A} / \mathrm{m}$ to the center of the Helmholtz cage. The supplied $\mathrm{H}$-field is recorded by measuring the voltage generated across a $50 \pm 0.05 \mathrm{~m} \Omega$ sense resistor. A general-purpose LM741 operation amplifier multiplies the sense resistor output by a factor of ten. Output from the op-amp is digitized by the Picoscope 2205A 8-bit ADC which is set to record a measurement once every $655.36 \mu \mathrm{s}$. In many applications, the ADC cannot communicate with the computer fast enough to enable real-time measurements. Instead, the Picoscope has onboard memory with a maximum capacity of 8000 measurements. This means that the Picoscope can measure about 5.25 cycles at $1 \mathrm{~Hz}$ before stopping to send the dataset to the computer. The Picoscope has two inputs which it can measure nearly simultaneously and has a triggering feature which ensures that all measurements begin at the same phase of the H-field cycle.

The sense coil output is multiplied by a dual INA2126 low-noise operational amplifier. The first amplification is a factor of 100, the second is a factor of two. After this amplification, the typical signal from the tested 
hysteresis rod given $\pm 100 \mathrm{~A} / \mathrm{m}$ is about $\pm 200 \mathrm{mV}$. This output is fed to the Picoscope ADC which digitizes the data for the computer.

\subsection{Method}

The hysteresis rod measurement method as performed by the user is outlined below.

1. Orient Helmholtz cage such that one axis is perpendicular to the local magnetic field.

2. Ensure no magnetic material is present in the Helmholtz cage test volume.

3. Place magnetometer in center of Helmholtz cage and align to Helmholtz cage axes (the magnetometer may be used to determine if the cage is properly aligned relative to the magnetic field).

4. Ensure that all axes of the Helmholtz cage are connected to the computercontrolled power supply outputs. The function generator / power amp output should not be connected yet.

5. Initialize the LabVIEW-based Helmholtz cage software. The software will now calculate the Helmholtz constant $\mathrm{HC}_{\text {empirical }}$.

6. Set the software to nullify the magnetic field on all three axes. If the Helmholtz cage is properly aligned, zero current should be needed to nullify the field on one of the axes.

7. Detach the power supply output from the coil set which is perpendicular to the B-field. Attach this coil set to the function generator / power amp output.

8. Enable the function generator at $1 \mathrm{~Hz}$ and enable the power amp. The magnetometer output should show varying magnetic field along a single axis.

9. Remove the magnetometer from the Helmholtz cage. Place the sense coil perpendicular to the zero-current coil set.

10. Set the function generator to the desired output voltage, being careful not to exceed $1.5 \mathrm{~V}$.

11. Collect one 8000-point dataset.

12. Place the magnetic sample within the sense coil being careful not to disturb the orientation of the coil.

13. Collect one 8000-point dataset.

14. Repeat steps 10-13 until all samples have been measured at all desired H-field amplitudes. 
The list above simply describes the physical process of collecting a hysteresis measurement; analysis occurs thereafter. Figure 7 shows a block diagram of the analysis process after collecting the digital dataset. The H-field is processed by removing the $10 \mathrm{x}$ amplification, converting to current, and multiplying by $\mathrm{HC}_{\text {empirical }}$. The B-field measurement is more complicated. After removing the gain of the signal, the signal due to the changing flux of the local environment is removed by subtracting the measurement without the sample from the measurement with the sample. After background subtraction, the data is numerically integrated using the trapezoidal method. The integrated voltage is converted to the B-field by application of Faraday's Law. The constant offset after numeric integration is determined by assuming that the hysteresis loop is symmetric; the constant offset of a linear fit to the hysteresis loop is removed from all B-field values.

The data is bifurcated into two distinct groups: data from the top/bottom hysteresis curves. The data grouping is based on whether the B-field is rising or falling (the sine wave behavior makes this easy to characterize). Further processing is possible due to the length of the each dataset (over five $\mathrm{H}$ field cycles) and the digitization of the $\mathrm{H}$-field measurements. The $\mathrm{H}$-field resolution of the 8-bit $\mathrm{ADC}$ varies from $0.105 \mathrm{~A} / \mathrm{m}( \pm 10 \mathrm{~A} / \mathrm{m})$ to $1.04 \mathrm{~A} / \mathrm{m}$ $( \pm 100 \mathrm{~A} / \mathrm{m})$. Thus, the measurements are further grouped into various Bfield values at each unique $\mathrm{H}$-field value and curve. The B-field average and standard deviation are calculated for the unique H-field values of each curve. This provides an estimate of the B-field uncertainty and is useful for further data processing.

\subsection{Results}

Three types of hysteresis measurements are performed: isolation, coupledhysteresis, and system flight-setup testing. Isolation testing measures hysteresis rod performance with no other magnetic materials nearby. Coupled hysteresis testing measures hysteresis rod performance when a second hysteresis rod is in close proximity; a variety of relative orientations and proximities are tested. System testing measures the hysteresis rod performance with a bar magnet and other hysteresis materials distributed identical to the flight satellite. In each measurement type, a nonlinear weighted leastsquares fit is used to determine the hysteresis parameters $\left(H_{c}, B_{r}\right.$, and $\left.B_{s}\right)$ which best match the data. The data are weighted by the inverse of their uncertainty and the Flatley hysteresis model [24] is used for the fit. Figure 8 

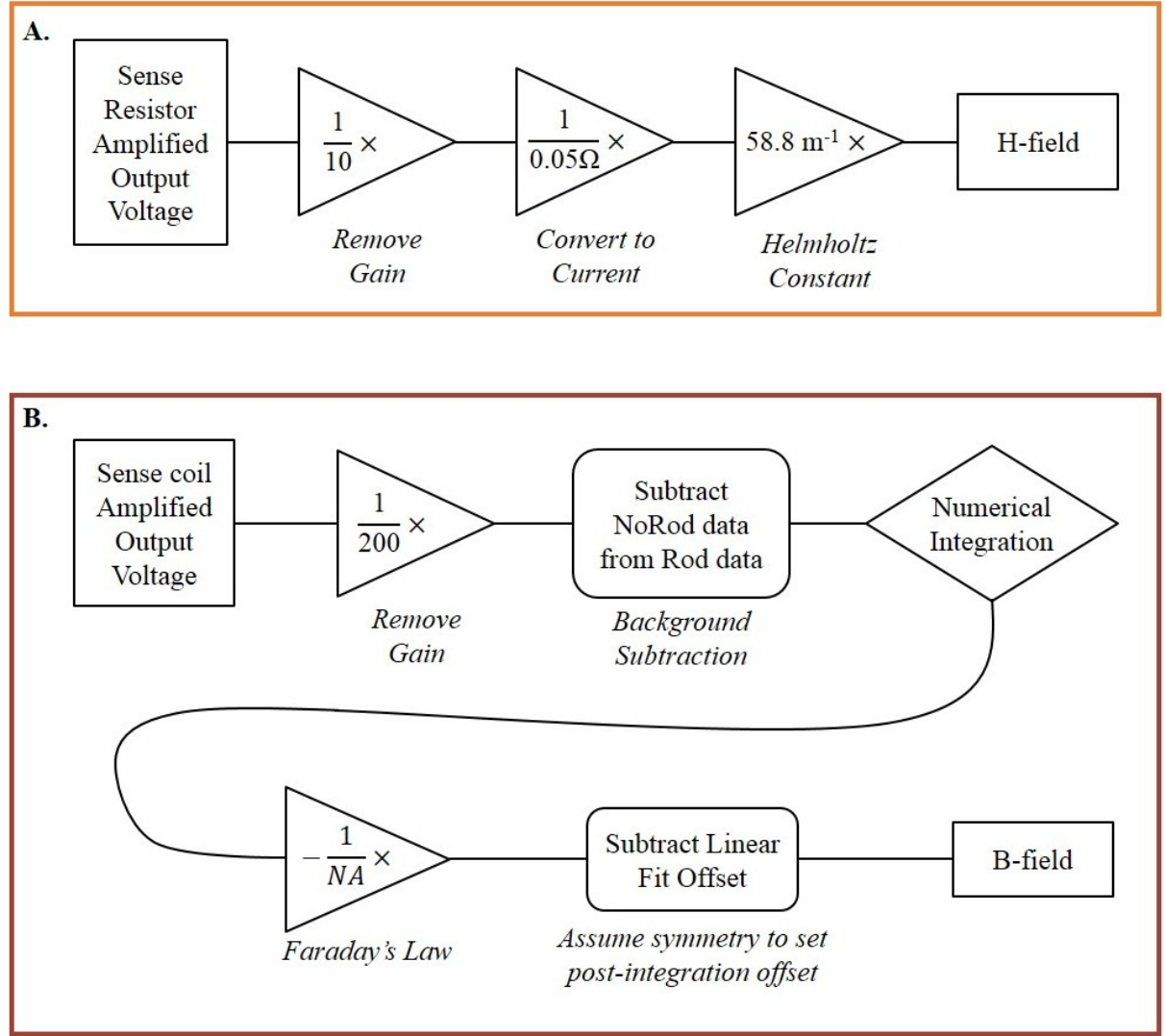

Figure 7: Hysteresis measurement analysis block diagram. The top (A.) and bottom (B.) rectangles highlight the analysis procedure for the $\mathrm{H}$-field and B-field, respectively. Multiplication is denoted by a triangle, subtraction is denoted by a rounded rectangle, and integration is denoted by a diamond. 


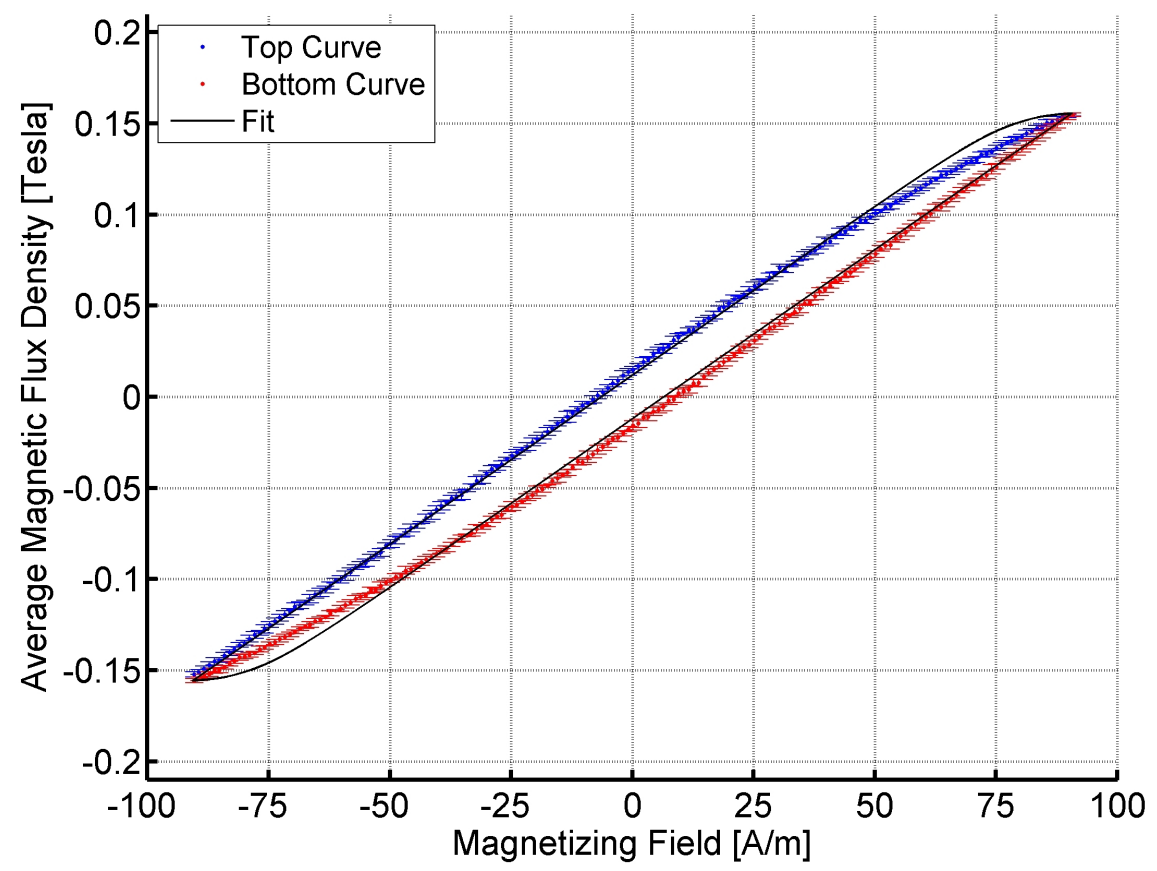

Figure 8: The $\pm 100 \mathrm{~A} / \mathrm{m}$ cycle amplitude measurement dataset of rod $\mathrm{B}$ with its fitted hysteresis loop. Error bars are included for each averaged flux density measurement.

shows one such $\pm 100 \mathrm{~A} / \mathrm{m}$ cycle amplitude dataset with its parameter-based fitted hysteresis loop.

Each experimental dataset was independently measured two times and independently fit to the Flatley model to develop an uncertainty estimate of the mean fit parameters. A small sample size correction to the standard error of the mean is applied to each of the uncertainty estimates [20]. Five CSSWE flight spare hysteresis rods were available for measurement (labeled rods A through E). The measurement is performed after the launch of CSSWE and uses hysteresis rods from the same raw material order and heat treatment set as the flight rods used on orbit.

\subsubsection{Isolated Measurement}

Each rod was isolated from other materials and cycled at five different H-field cycle amplitudes: $\pm 10 \mathrm{~A} / \mathrm{m}, \pm 20 \mathrm{~A} / \mathrm{m}, \pm 30 \mathrm{~A} / \mathrm{m}, \pm 50 \mathrm{~A} / \mathrm{m}$, and $\pm 100 \mathrm{~A} / \mathrm{m}$. The Flatley model was then used to fit hysteresis parameters 


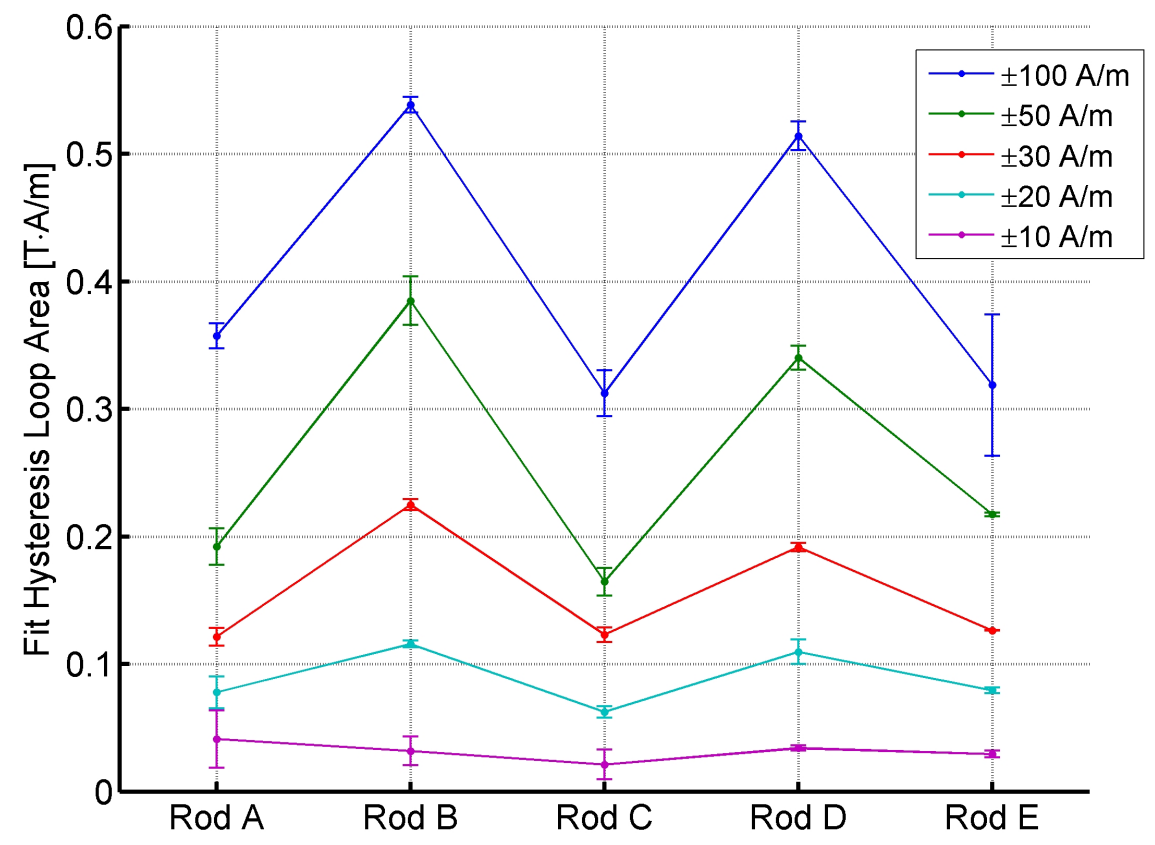

Figure 9: The simulated $\pm 20 \mathrm{~A} / \mathrm{m}$ hysteresis loop area for hysteresis parameters fitted to a variety of $\mathrm{H}$-field cycle amplitude datasets gathered for each of five measurement hysteresis rods. Each measurement was recorded with no other magnetic materials nearby.

$\left(H_{c}, B_{r}\right.$, and $\left.B_{s}\right)$ to each dataset. Finally, the fitted parameters are used in conjunction with the Flatley model to determine the area enclosed by the hysteresis loop given a $\pm 20 \mathrm{~A} / \mathrm{m}$ simulated cycle magnitude.

The $\pm 20 \mathrm{~A} / \mathrm{m}$ area for each rod and empirical cycle magnitude is shown in Figure 9. Note that the product of the hysteresis loop area and the hysteresis rod volume is the energy loss per H-field cycle. Thus, the dampening ability of each rod varies significantly. Surprisingly, Figure 9 shows that the simulated $\pm 20 \mathrm{~A} / \mathrm{m}$ loop area of each fit also varies significantly depending on the $\mathrm{H}$-field cycle amplitude during the empirical measurement. If the hysteresis model behaved perfectly, fit parameters based on a variety of empirical $\mathrm{H}$-field cycle amplitudes would yield identical simulated cycle loop areas at the same simulated cycle amplitude.

Figures 10 and 11 compare the hysteresis loops generated from a variety 
of simulated cycle amplitudes given hysteresis parameters fit data measured from \pm 100 and $\pm 10 \mathrm{~A} / \mathrm{m}$ empirical cycle amplitudes, respectively. The simulated loop areas for each fit at identical simulated cycle amplitudes are quite different. The fitted parameters best represent the true hysteresis loop when the measurement magnetizing field cycle amplitude is close to the simulated cycle amplitude. Thus, when using the Flatley model, to achieve accurate simulation results, the measurement cycle amplitude should equal the range of magnetizing field which the true hysteresis rod is expected to experience the most frequently during dampening.

The extremes of the magnetization cycle amplitude are bounded by the magnitude of the on-orbit H-field but the extremes of the H-field component parallel to each rod will vary as the satellite attitude settles. Figure 12 shows the magnetizing field parallel to the hysteresis rods mounted along the ${ }^{\mathcal{B}} X$ and ${ }^{\mathcal{B}} Y$ axes. As shown, CSSWE experienced magnetizing field cycle amplitudes from $\pm 40 \mathrm{~A} / \mathrm{m}$ to less than $\pm 5 \mathrm{~A} / \mathrm{m}$ within the first seven days after launch. Note that smaller cycles due non-flat-spin motion occur but are difficult to see in this plot. For this reason, the fitted hysteresis parameters used by the simulation are based on measurement cycle amplitudes of \pm 20 and $\pm 10 \mathrm{~A} / \mathrm{m}$; these values are chosen in an attempt to use the most frequent cycle amplitudes experienced by each hysteresis rod during the settling period.

Figures 10 and 11 also show that the $\pm 100 \mathrm{~A} / \mathrm{m}$ model degrades at especially small cycle amplitudes: each cycle is no longer a closed loop. This behavior is unrealistic and undesired. The parameters from the $\pm 10 \mathrm{~A} / \mathrm{m}$ cycle perform much better at these low magnetizing field levels. Correctly representing these small cycle amplitudes is important because these loops are regularly encountered due to non-flat-spin motion throughout dampening. This is another reason to use a low cycle amplitude during measurement, though it requires a more sensitive measurement system.

\subsubsection{Coupled Hysteresis Measurement}

PMAC dampening ability could be degraded if hysteresis rods are placed too close together in certain orientations [30]. The Helmholtz cage and sense coil setup is uniquely able to test which relative geometries degrade performance and which do not. Figure 13 shows the performance of rod A (placed within the sense coil, parallel to the H-field forcing) when Rod E (outside the sense coil, perpendicular to H-field forcing) is oriented such that the two rods form a $\mathrm{T}$ or $\mathrm{L}$ shape. Rods $\mathrm{A}$ and $\mathrm{E}$ are used because these rods were 

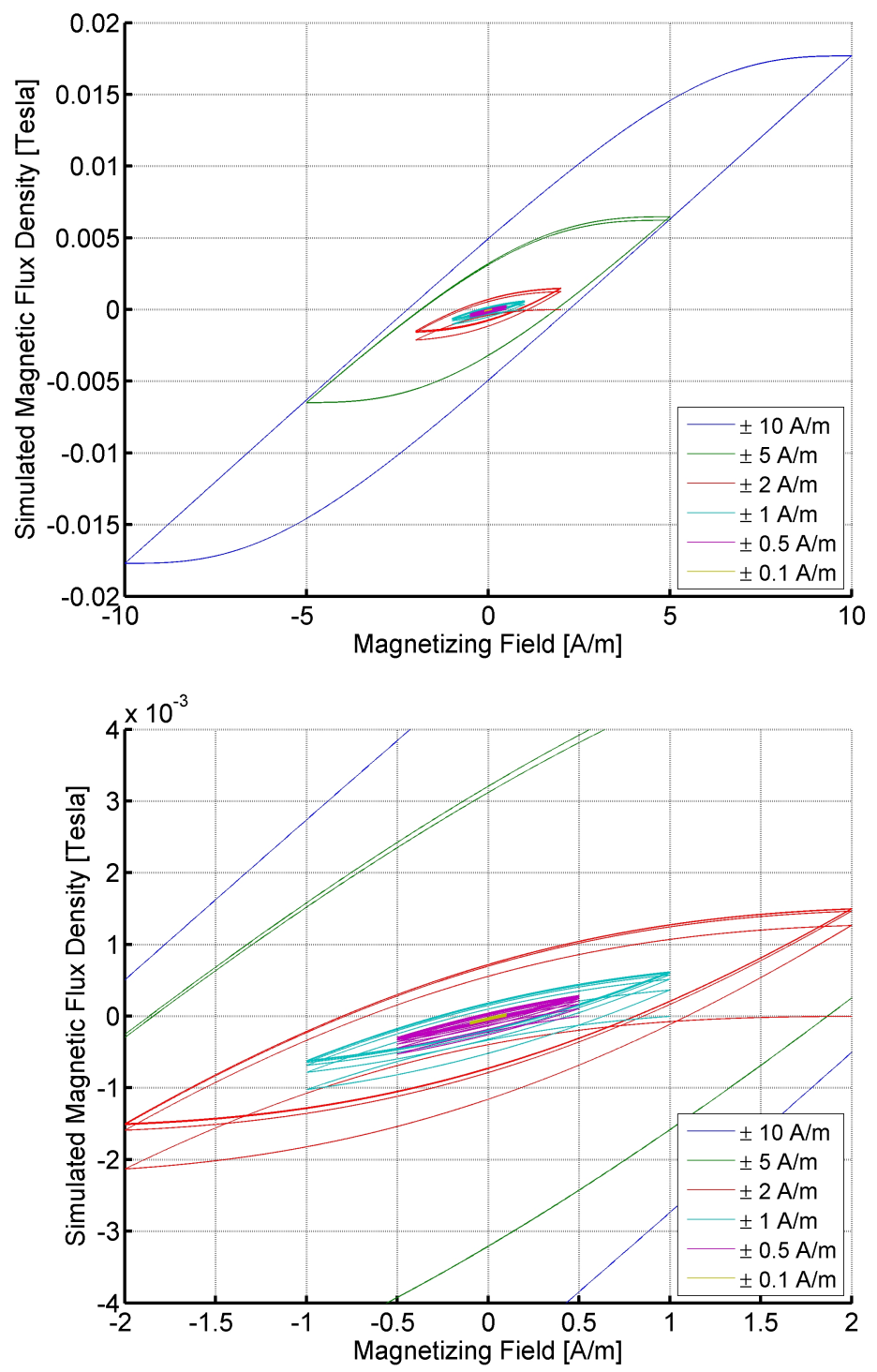

Figure 10: A variety of simulated hysteresis loops generated using parameters fitted to measured output from an empirical cycle amplitude of $\pm 100 \mathrm{~A} / \mathrm{m}$. Each H-field amplitude is used to simulate 10 cycles of data. The top plot shows the simulated performance at bounds of $\pm 10 \mathrm{~A} / \mathrm{m}$; the bottom plot zooms to show the same data at a range of $\pm 2 \mathrm{~A} / \mathrm{m}$. 

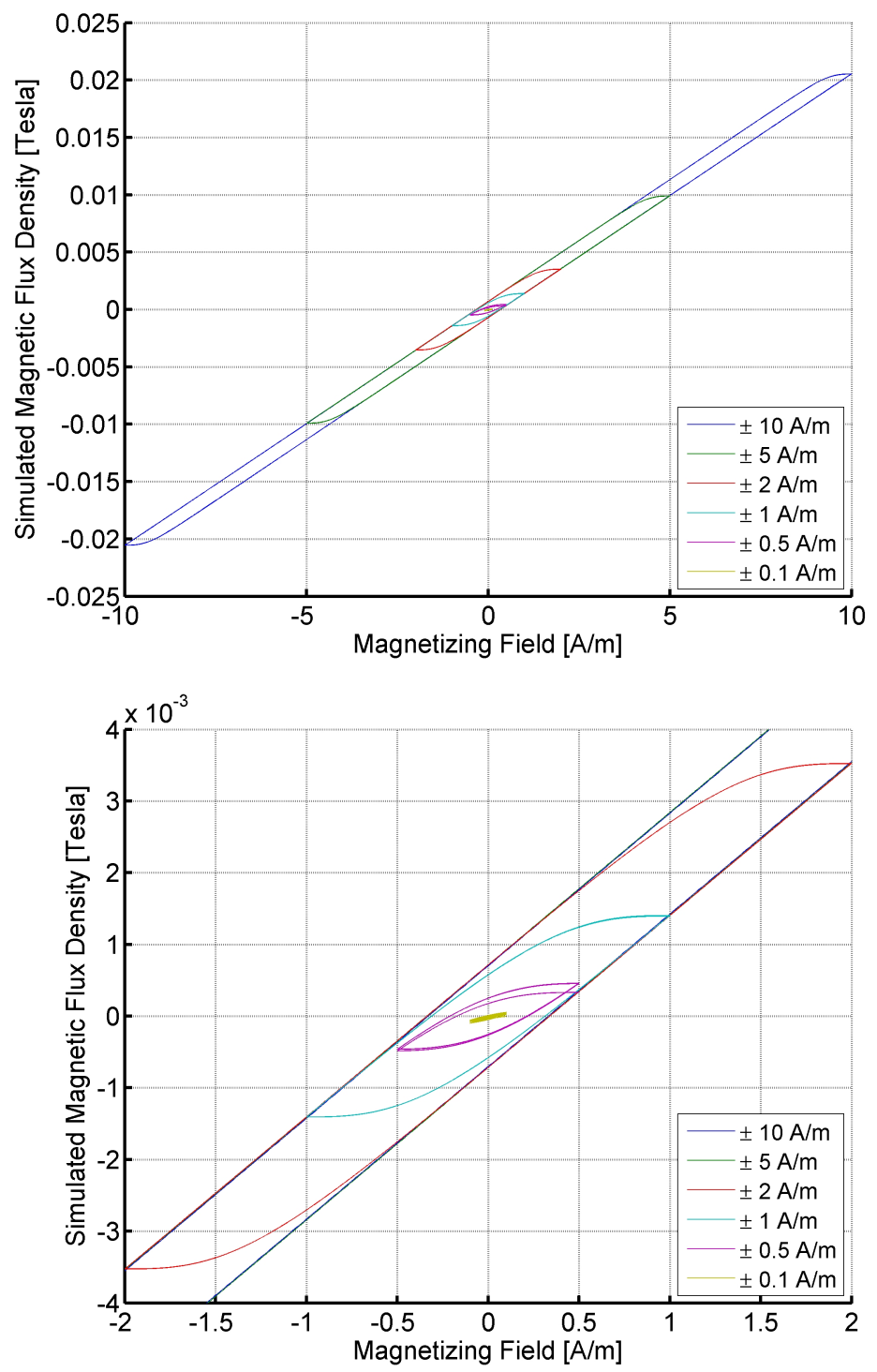

Figure 11: A variety of simulated hysteresis loops generated using parameters fitted to measured output from an empirical cycle amplitude of $\pm 10 \mathrm{~A} / \mathrm{m}$. Each H-field amplitude is used to simulate 10 cycles of data. The top plot shows the simulated performance at bounds of $\pm 10 \mathrm{~A} / \mathrm{m}$; the bottom plot zooms to show the same data at a range of $\pm 2 \mathrm{~A} / \mathrm{m}$. 

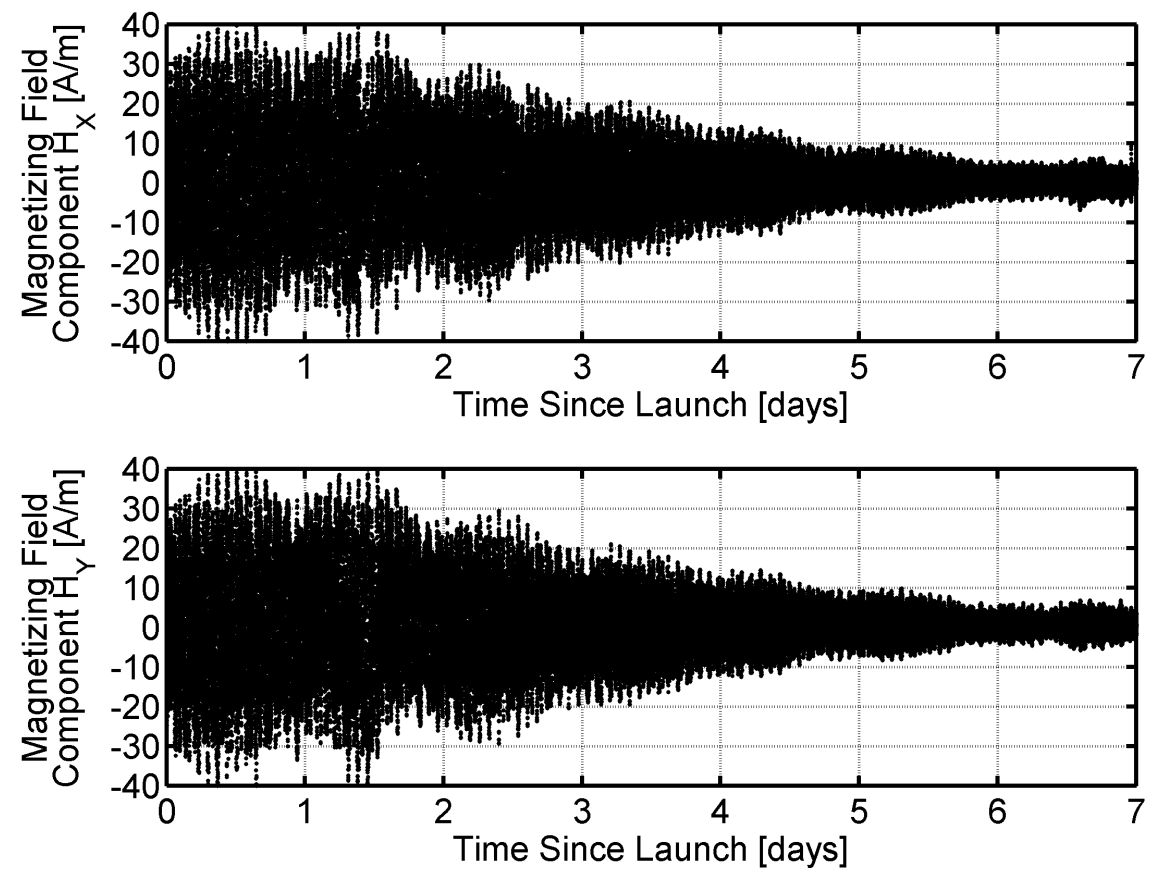

Figure 12: The magnetizing field experienced by each of the body-frame axes with parallel hysteresis rods $\left({ }^{\mathcal{B}} X\right.$ and $\left.{ }^{\mathcal{B}} Y\right)$ over the first week on-orbit. Each hysteresis rod experiences a wide variety of $\mathrm{H}$-field cycle amplitudes. 


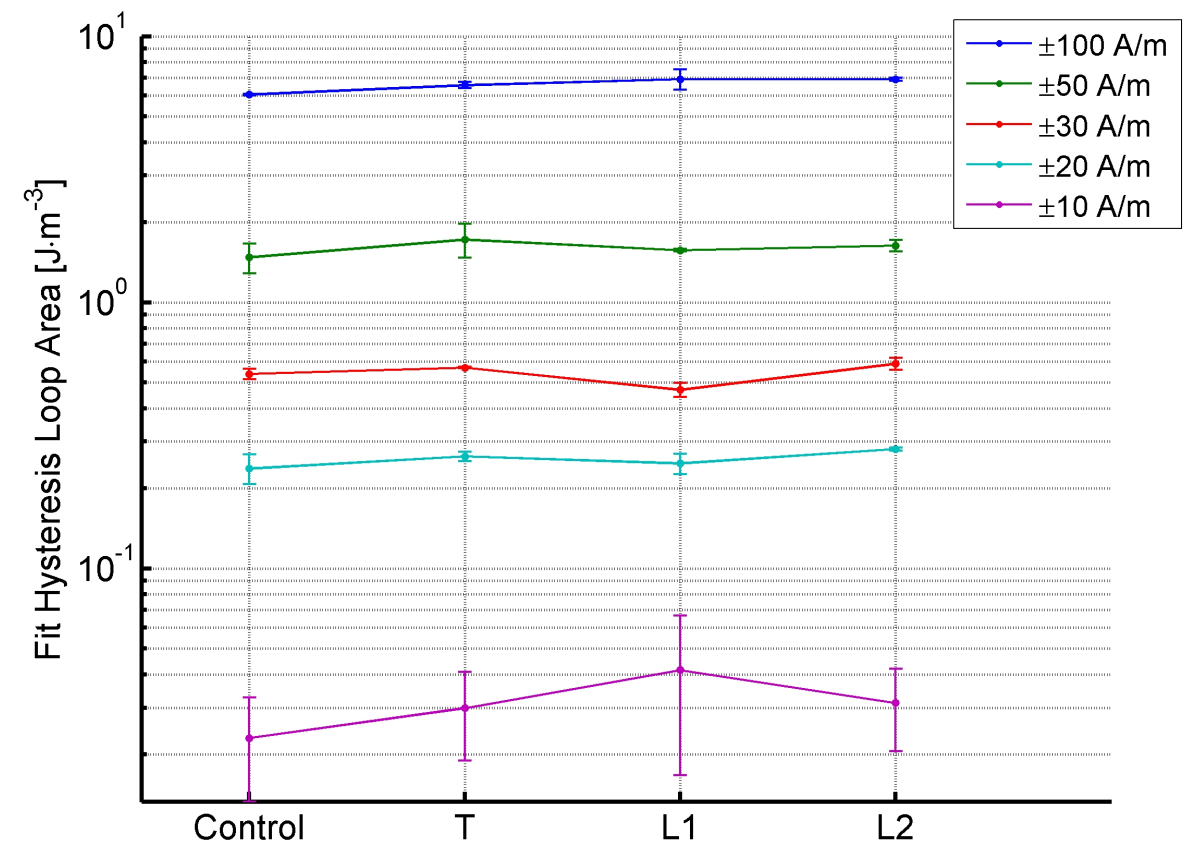

Figure 13: The simulated $\pm 20 \mathrm{~A} / \mathrm{m}$ hysteresis loop area for hysteresis parameters fitted to a variety of $\mathrm{H}$-field cycle amplitude datasets gathered for multiple perpendicular relative geometries. Rod A was placed in the sense coil and Rod E was placed perpendicular to the sense coil. The "T" configuration puts the intersection of Rod A and E halfway through Rod E. The "L1" configuration has Rod A in the same orientation, but slides Rod E until the intersection is at the end of each rod. The "L2" configuration puts the rod intersection point at the opposite end of Rod E.

previously found to have similar magnetization levels (Figure 9). The T- and L-shapes are tested because many PMAC system designs use this layout, especially in CubeSats. A control measurement (with no rod E present) is also shown for comparison. As shown in Figure 13, the dampening ability of rod A is not significantly impacted by the presence of a perpendicularly-oriented rod E, regardless of the relative geometry.

Figure 14 shows the performance of rod A when rod E is parallel (and thus also magnetized by H-field cycling) at a variety of distances between the rods ( $5 \mathrm{~mm}$ to $50 \mathrm{~mm}$ separation). Again, the control without rod $\mathrm{E}$ is also shown for comparison. Here there is a significant degradation in dampening ability 


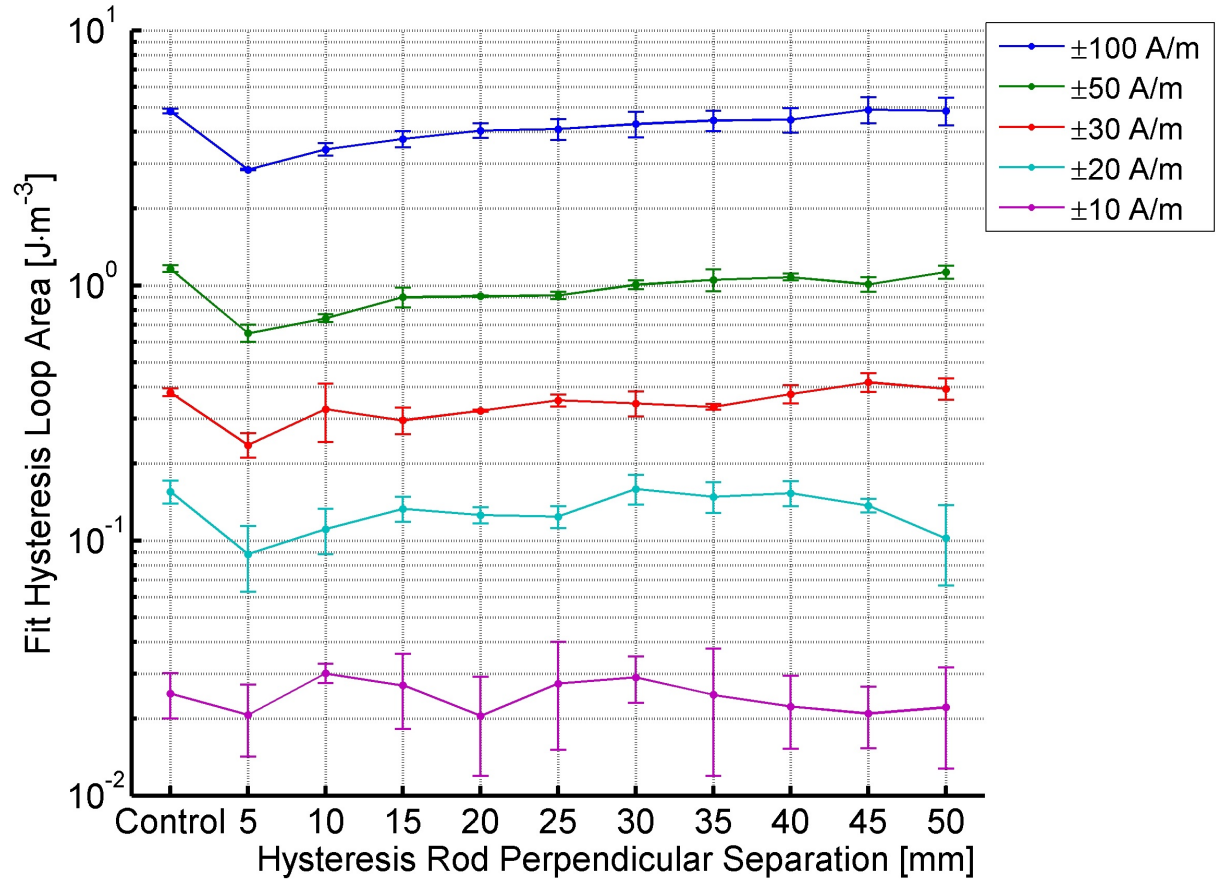

Figure 14: The simulated $\pm 20 \mathrm{~A} / \mathrm{m}$ hysteresis loop area for hysteresis parameters fitted to a variety of $\mathrm{H}$-field cycle amplitude datasets gathered for parallel relative geometries at a variety of distances. Rod A was placed in the sense coil and Rod E was placed outside and parallel to the sense coil. Rods A and E are separated by distances of $5 \mathrm{~mm}$ to $50 \mathrm{~mm}$.

when the rod separation is less than $30 \%$ to $40 \%$ of the rod length (each rod is $9.5 \mathrm{~cm}$ ), which is in agreement with a general PMAC heuristic [30]. This measurement is also in good agreement with Figure 9 of [27], which shows that groupings of thin $100 \mathrm{~mm}$ length hysteresis strips have an exponentially increasing global demagnetization factor when grouped closer than $40 \mathrm{~mm}$ together.

\subsubsection{Flight Setup Hysteresis Measurement}

The layout of PMAC components within a satellite impacts the dampening ability of each hysteresis rod. The net effect of the layout may be measured by placing magnetic components in their relative satellite positions and measuring individual hysteresis rod performance. Figure 15 shows a solid model of the CSSWE CubeSat with the positions of the hysteresis 
rods and the bar magnet highlighted in red.

Hysteresis rods B, C, D, and E were placed at the X2, X3, Y2, and Y3 hysteresis rod positions within a CSSWE flight structure mock-up (see Figure 15). A flight spare bar magnet was also added to the mock-up in the flight position. The sense coil was then used to measure the performance of rod A at each of the hysteresis rod positions; the native rod at each position was removed during the measurement. Figure 16 shows the full setup for this measurement while Figure 17 shows the simulated area of the fitted hysteresis parameters.

There is some variation but the difference is only significant for the \pm 100 $\mathrm{A} / \mathrm{m}$ cycle amplitudes case. The hysteresis rod positions closest to the bar magnet (X3 and Y3) could be expected to possess decreased dampening abilities due to their increased H-field offset. The data shows that, for the CSSWE layout, interactions between rods are more important than the offset due to the bar magnet. Figure 18 shows the calculated H-field parallel to each rod position due to an $0.80 \mathrm{~A} \cdot \mathrm{m}^{2}$ dipole aligned with $+{ }^{\mathcal{B}} Z$ at the bar magnet location. Although the rods each experience a magnetizing field variation of up to $4.5 \mathrm{~A} / \mathrm{m}$ along their length, the average $\mathrm{H}$-field offset is $1-2 \mathrm{~A} / \mathrm{m}$.

\subsubsection{Best-Fit Hysteresis Parameters}

The individual testing shows that the hysteresis rod dampening ability varies significantly for each individual rod. However, the system testing shows that the position of a given rod within the CSSWE layout does not have a significant effect on the dampening ability of the rod at cycle amplitudes less than $\pm 50 \mathrm{~A} / \mathrm{m}$; Figure 12 shows that the CSSWE hysteresis rods experience H-fields amplitudes of less than $|40| \mathrm{A} / \mathrm{m}$. Also, the hysteresis model has been found to work best when simulating data closest to its measurement cycle amplitude. To simplify the simulation, one set of parameters is used to model all six hysteresis rods within CSSWE. With all of the above considerations in mind, the best-fit parameters are found by fitting the individual measurement data from all five hysteresis rods over both tests at magnetizing field cycle amplitudes of both \pm 10 and $\pm 20 \mathrm{~A} / \mathrm{m}$.

The fitted hysteresis loop parameters are collected in Table 2 and compared to the closed magnetic circuit values listed for the rod material. Note that the datasheet-based hysteresis parameters yield a loop area nearly $100 \times$ greater than the measurement-based values. This difference in hysteresis dampening ability will have the profound effect of changing the simulated settling time by the same factor. Unfortunately, this error leads to unrealistically- 


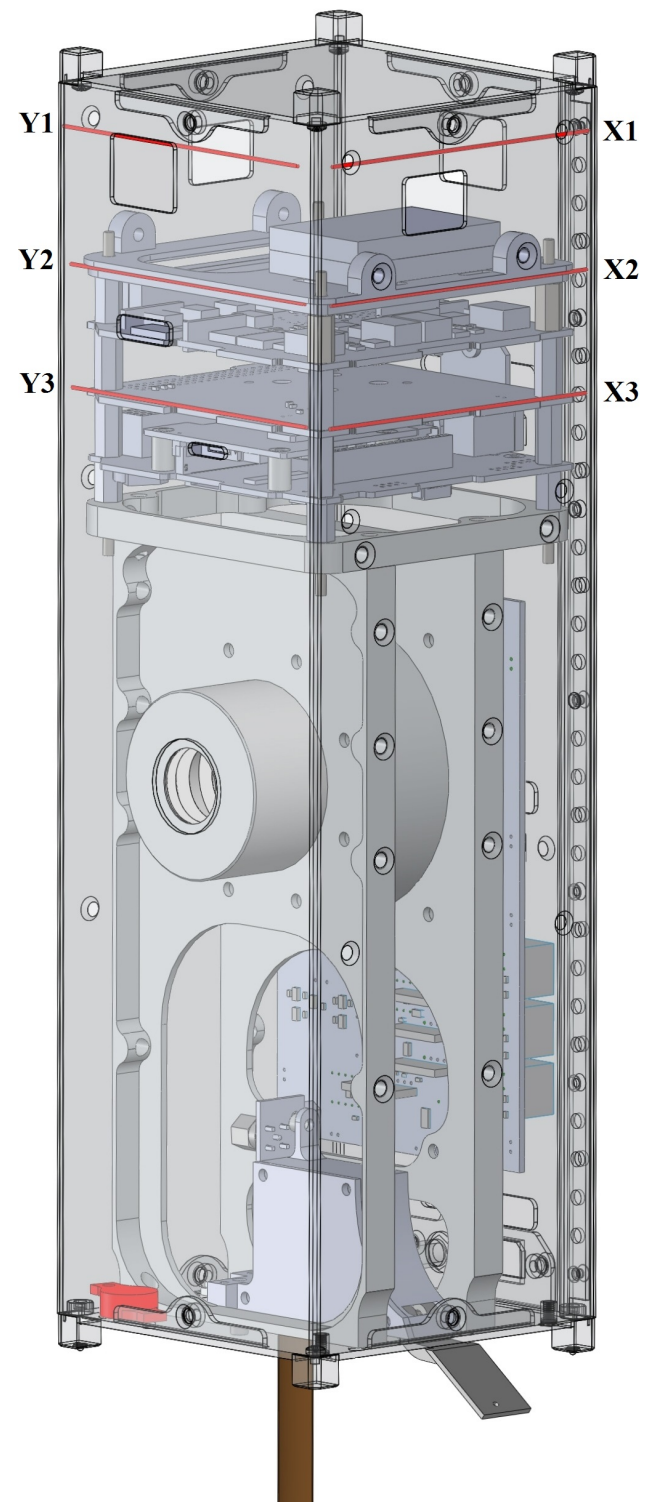

Figure 15: A solid model highlighting the position of PMAC components. The hysteresis rods (top) have a large separation from the bar magnet (bottom left) in order to prevent magnetic offsets to the rod hysteresis loops. Each hysteresis rod position is labeled; the rod sets are separated by a minimum perpendicular distance of $3.25 \mathrm{~cm}$. 


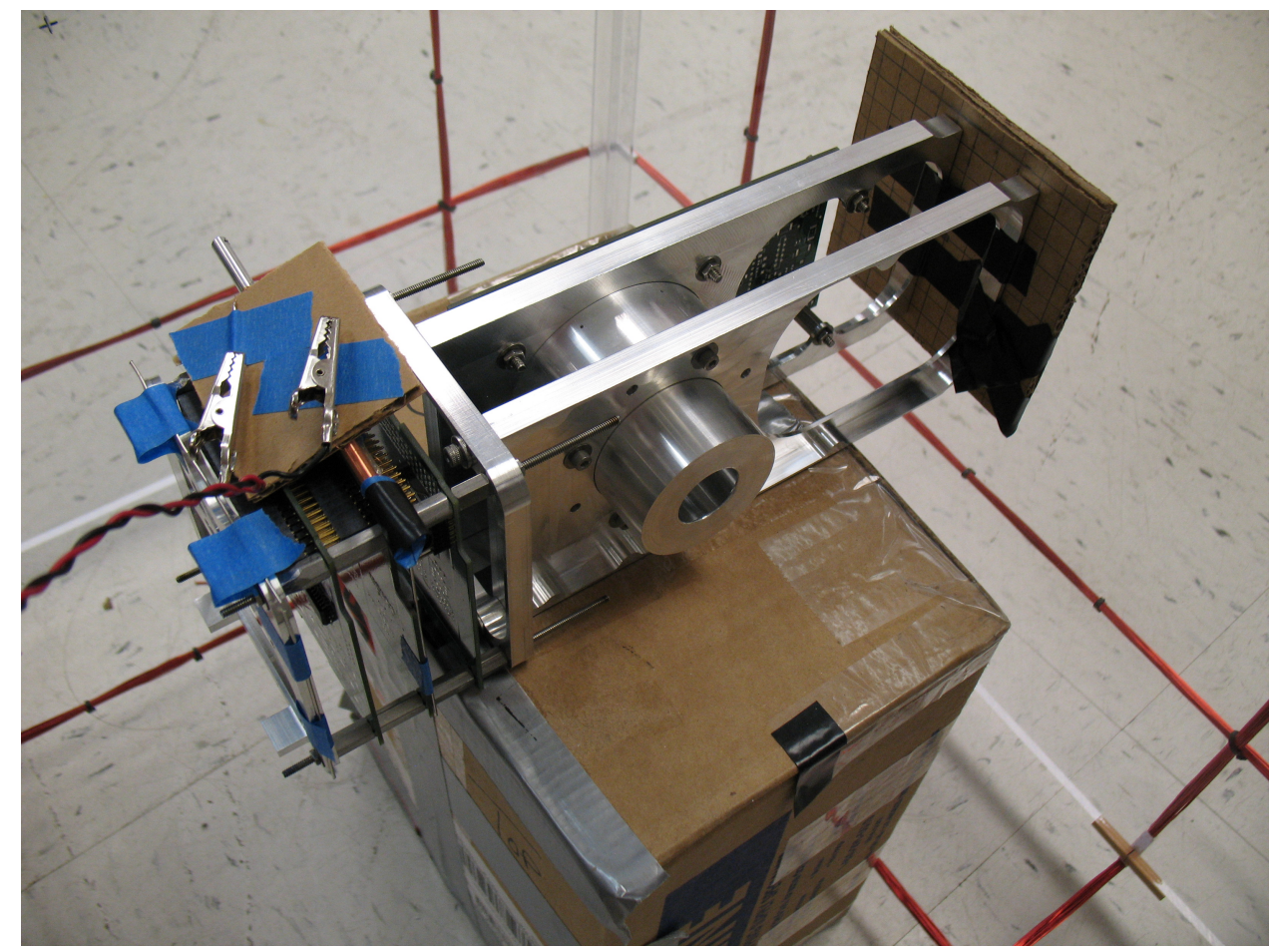

Figure 16: The CSSWE flight structure mock-up with hysteresis rods and bar magnet attached is used to measure the effect of other magnetic sources on a single hysteresis rod. The sense coil is in the X3 position (as defined by Figure 15). 


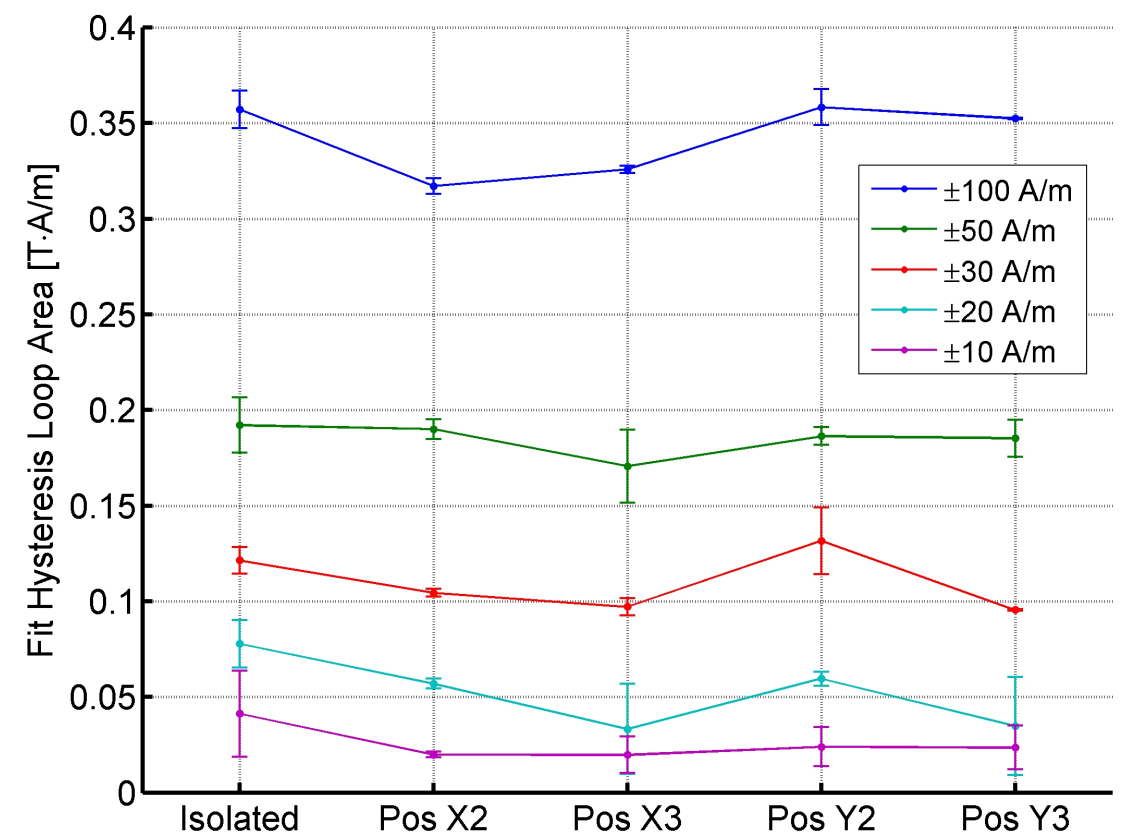

Figure 17: The simulated hysteresis loop area using hysteresis parameters fitted to Rod A measurements performed at a variety of cycle amplitudes. The measurements are collected at each of the X2, X3, Y2, and Y3 hysteresis positions labeled in Figure 15. The Rod A Isolated Testing results are repeated here for comparison. 


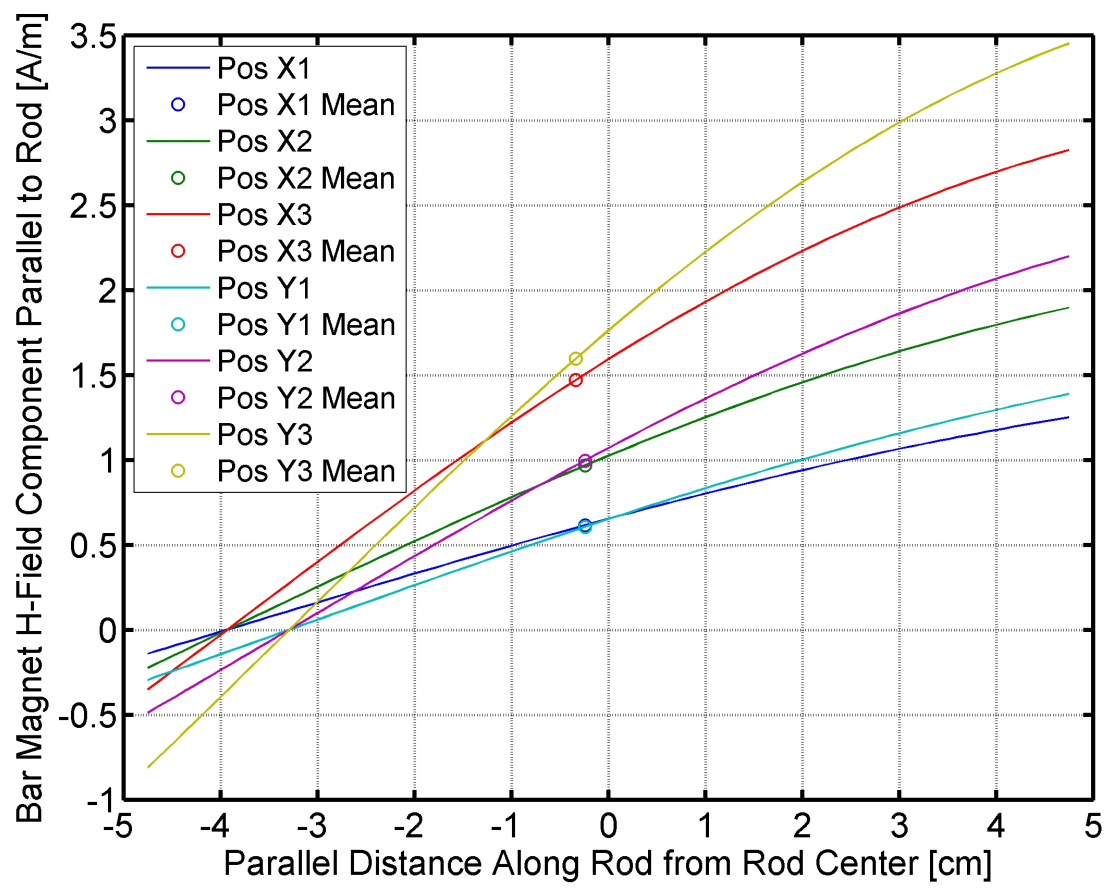

Figure 18: The magnetizing field offset parallel to each hysteresis rod position due to an $0.80 \mathrm{~A} \cdot \mathrm{m}^{2}$ dipole aligned with $+{ }^{\mathcal{B}} Z$ at the bar magnet location. The value of the mean $\mathrm{H}$-field parallel to each rod is shown using open circles. 
Table 2: HyMu-80 Hysteresis Parameters. The area was calculated using the Flatley hysteresis model [24] with a cycle amplitude of $\pm 20 \mathrm{~A} / \mathrm{m}$.

\begin{tabular}{lll}
\hline Hysteresis Parameter & $\begin{array}{l}\text { Closed Magnetic } \\
\text { Circuit Value } \\
\text { (Material Datasheet) }\end{array}$ & $\begin{array}{l}\text { Open Magnetic } \\
\text { Circuit Value } \\
\text { (Fitted to Measurement) }\end{array}$ \\
\hline$H_{c}(\mathrm{~A} / \mathrm{m})$ & 1.59 & 0.3381 \\
$B_{r}($ Tesla $)$ & 0.35 & $6.0618 \times 10^{-4}$ \\
$B_{s}$ (Tesla) & 0.73 & 0.3000 \\
Loop Area $\left(\mathrm{J} \cdot \mathrm{m}^{-3}\right)$ & 4.312 & 0.0448 \\
\hline
\end{tabular}

short settling times. A designer simulating hysteresis performance using these incorrect parameters may not include enough dampening material within the spacecraft, leading to settling times on the order of months rather than days. Such behavior has been observed multiple times [12] [13] and stresses the importance of hysteresis measurement within any PMAC system.

\section{Conclusions}

The importance and benefits of hysteresis measurement have been illustrated above. First, a Helmholtz cage was designed to control the magnetic field with a variation of $1 \%$ within a $30 \times 30 \times 30 \mathrm{~cm}$ volume. This cage was built for less than $\$ 1500$ and served as the core of the magnetic measurement testbed. Post-build testing showed that the variation over the test volume was $5 \%$, but this is attributed to magnetic field gradients inherent to the local environment. The cage was used to determine that the CSSWE hard magnet possesses a magnetic dipole moment of $0.80 \pm 0.017 \mathrm{~A} \cdot \mathrm{m}^{2}$. This is a key input to any PMAC simulation and served as a sanity check for the further use of the cage.

Magnetization of the entire Helmholtz cage test volume was used to ensure that the hysteresis measurements accurately reflected the in-orbit environment of CSSWE. Parameter fitting to multiple isolated hysteresis rods resulted in two key findings. First, there is significant variation among the individual CSSWE flight-spare hysteresis rods, even through they originated from the same lot. Second, the Flatley model cannot accurately portray the non-linear variations of dampening performance over a wide range of magnetizing field cycle amplitudes. If it is to be used for simulation purposes, a 
single hysteresis parameter set should be measured using H-field cycle amplitudes close to the most-frequently expected on-orbit cycle magnitudes.

The volume magnetization of the Helmholtz cage is used to investigate the relationship between two hysteresis rods: first when perpendicular to one another and second when parallel. In the perpendicular case, we found no significant change in dampening performance. This validates the relative placement of hysteresis rods used within many nanosatellites. In the parallel case, the nearby rod is found to have a significant impact when the separation is less than $30 \%$ to $40 \%$ of the rod length. Finally, the true arrangement of magnetic materials used within the CSSWE satellite is replicated; no significant impact on the fitted hysteresis parameters is observed (compared to the individual rod case). This validates the PMAC design used aboard CSSWE; it does not imply that volume-based magnetization is not needed in the general case.

The hysteresis rod parameters fitted to the CSSWE mission are based on cycle amplitudes of both $\pm 10 \mathrm{~A} / \mathrm{m}$ and $\pm 20 \mathrm{~A} / \mathrm{m}$ as these are what the satellite saw most frequently in the early mission. The fitted parameters result in $100 \times$ less dampening as compared to the closed magnetic circuit parameters. This underlines the importance of hysteresis measurement for accurate simulation of PMAC performance.

\section{Acknowledgements}

The authors are indebted to the students and professionals who contributed to the CSSWE satellite, helping move it from conception to orbit. Foremost among them is Professor Xinlin Li, who led the CSSWE team over many years to achieve mission success. Thanks also to Tony Kos at the National Institute of Standards and Technology who provided invaluable support throughout the development of the measurement setup. This research

was supported by the National Science Foundation grants ATM-0940277 and AGS-1242893.

[1] R. J. Danchik, An Overview of Transit Development, John Hopkins APL Technical Digest 19 (1) (1998) 18-26.

[2] S. Rawashdeh, Passive Attitude Stabilization for Small Satellites, University of Kentucky Masters Thesis. 
[3] F. Santoni, M. Zelli, Passive magnetic attitude stabilization of the unisat-4 microsatellite, Acta Astronautica 65 (2009) 792-803.

[4] G. Minelli, C. Kitts, K. Ronzano, C. Beasley, R. Rasay, I. Mas, P. Williams, P. Mahacek, J. Shepard, J. Acain, J. Hines, E. Agasid, C. Friedericks, M. Piccini, M. Parra, L. Timucin, M. Henschke, E. Luzzi, N. Mai, M. Mcintyre, R. Ricks, D. Squires, C. Storment, J. Tucker, B. Yost, G. Defouw, A. Ricco, Extended Life Flight Results from the GeneSat-1 Biological Microsatellite Mission, in: USU Conference on Small Satellites, 2008, pp. 1-10. doi:SSC08-II-4.

[5] J. W. Cutler, H. Bahcivan, Radio Aurora Explorer: A Mission Overview, Journal of Spacecraft and Rockets (2013) 1-9doi:10.2514/1.A32436.

URL http://arc .aiaa.org/doi/abs/10.2514/1.A32436

[6] S. Palo, Conducting science with a cubesat: the colorado student space weather experiment, in: Small Satellite Conference, no. SSC10-XII-8, AIAA/USU, 2010.

[7] X. Li, S. Palo, R. Kohnert, Small Space Weather Research Mission Designed Fully by Students, Space Weather 9 (4). doi:10.1029/2011SW000668.

URL http://www .agu .org/pubs/crossref/2011/2011SW000668.shtml

[8] X. Li, S. Palo, R. Kohnert, L. Blum, D. Gerhardt, Q. Schiller, S. Califf, Small Mission Accomplished by Students-Big Impact on Space Weather Research, Space Weather 11 (2) (2013) 55-56. doi:10.1002/swe.20025. URL http://doi.wiley.com/10.1002/swe. 20025

[9] G. L. Skrobot, ELaNa Educational Launch of Nanosatellite Enhance Education through Space Flight, in: Small Sat Conference, 2011, pp. SSC11-II-2.

[10] G. L. Skrobot, R. Coelho, ELaNa - Educational Launch of Nanosatellite: Providing Routine RideShare Opportunities, in: Small Satellite Conference, 2012.

[11] D. Gerhardt, S. E. Palo, Q. Schiller, L. Blum, X. Li, R. Kohnert, The Colorado Student Space Weather Experiment (CSSWE) On-Orbit Performance, Journal of Small Satellites 3 (1) (2014) 265-281. 
[12] J. C. Springmann, B. P. Kempke, J. W. Cutler, Initial Flight Results of the RAX-2 Satellite, in: Small Sat Conference, 2012.

[13] R. J. Hamann, J. Bouwmeester, G. Brouwer, Delfi-C3 Preliminary Mission Results, in: Small Satellite Conference, 2009, pp. SSC09-IV-7.

[14] A. E. Ruark, M. F. Peters, Helmholtz Coils for Producing Uniform Magnetic Fields, Journal of the Optical Society of America 13 (175) (1926) 205-212. doi:10.1364/JOSA.13.000205.

[15] A. Klesh, S. Seagraves, M. Bennett, D. Boone, J. Cutler, Dynamically Driven Helmholtz Cage for Experimental Magnetic Attitude Determination, Advances In Astronautical Sciences 135 (suppl. 1) (2009) 147-160.

[16] F. Piergentili, G. Candini, M. Zannoni, Design, Manufacturing, and Test of a Real-Time, Three-Axis Magnetic Field Simulator, IEEE Transactions on Aerospace and Electronic Systems 47 (2) (2011) 1369-1379. doi:10.1109/TAES.2011.5751264.

[17] California Polytechnic State University, CubeSat Design Specification, rev. 12.

[18] C. C. Finlay, S. Maus, C. D. Beggan, T. N. Bondar, A. Chambodut, T. A. Chernova, A. Chulliat, V. P. Golovkov, B. Hamilton, M. Hamoudi, R. Holme, G. Hulot, W. Kuang, B. Langlais, V. Lesur, F. J. Lowes, H. Lühr, S. Macmillan, M. Mandea, S. McLean, C. Manoj, M. Menvielle, I. Michaelis, N. Olsen, J. Rauberg, M. Rother, T. J. Sabaka, a. Tangborn, L. Tø ffner Clausen, E. Thébault, A. W. P. Thomson, I. Wardinski, Z. Wei, T. I. Zvereva, International Geomagnetic Reference Field: the eleventh generation, Geophysical Journal International 183 (3) (2010) 1216-1230. doi:10.1111/j.1365-246X.2010.04804.x. URL http://doi.wiley.com/10.1111/j.1365-246X.2010.04804.x

[19] G. W. Prölss, Physics of the Earth's Space Environment, 1st Edition, Springer Press, 2004.

[20] J. Gurland, R. Tripathi, A Simple Approximation for Unbiased Estimation of the Standard Deviation, The American Statistician 25 (4) (1971) $30-32$. 
[21] B. Cullity, C. Graham, Introduction to Magnetic Materials, 2nd Edition, Wiley-IEEE Press, 2008.

[22] J.-F. Levesque, Passive magnetic attitude stabilization using hysteresis materials, Tech. Rep. SIgMA-PU-006-UdeS, Université de Sherbrooke (1995).

[23] G. Park, S. Seagraves, N. H. McClamroch, A Dynamic Model of a Passive Magnetic Attitude Control System for the RAX Nanosatellite, in: AIAA Guiidance, Navigation and Control Conference, no. August, 2010.

[24] T. W. Flatley, D. A. Henretty, A magnetic hysteresis model, Tech. Rep. N95-27801, NASA GSFC (1995).

[25] D. S. Ivanov, M. Y. Ovchinnikov, V. I. Penkov, Laboratory study of magnetic properties of hysteresis rods for attitude control systems of minisatellites, Journal of Computer and Systems Sciences International 52 (1) (2013) 145-164. doi:10.1134/S1064230712060032.

[26] M. L. Battagliere, F. Santoni, M. Y. Ovchinnikov, F. Graziani, Hysteresis Rods In the Passive Magnetic Stabilization System for University Micro and Nanosatellites, in: 59th International Astronautical Congress, 2008, pp. 5203-5212.

[27] F. Santoni, M. L. Battagliere, F. Fiorillo, E. Ferrara, Optimal Geometry and Materials for Nanospacecraft Magnetic Damping Systems, IEEE Transactions on Aerospace and Electronic Systems 51 (1) (2015) 127141.

[28] M. L. Battagliere, F. Santoni, F. Piergentili, M. Ovchinnikov, F. Graziani, Passive magnetic attitude stabilization system of the EduSAT microsatellite, Proceedings of the Institution of Mechanical Engineers, Part G: Journal of Aerospace Engineering 224 (10) (2010) 1097-1106. doi:10.1243/09544100JAERO732.

[29] F. Fiorillo, F. Santoni, E. Ferrara, M. L. Battagliere, O. Bottauscio, F. Graziani, Soft Magnets for Passive Attitude Stabilization of Small Satellites, IEEE Transactions on Magnetics 46 (2) (2010) 670-673. doi:10.1109/TMAG.2009.2033345. 
[30] M. Y. Ovchinnikov, V. Penkov, Passive magnetic attitude control system for the munin nanosatellite, Cosmic Research 40 (2) (2002) 142-156. 